\title{
ANALYSIS OF A SEMI-LAGRANGIAN METHOD FOR THE SPHERICALLY SYMMETRIC VLASOV-EINSTEIN SYSTEM*
}

\author{
Philippe Bechouche ${ }^{1}$ And Nicolas Besse ${ }^{2}$
}

\begin{abstract}
We consider the spherically symmetric Vlasov-Einstein system in the case of asymptotically flat spacetimes. From the physical point of view this system of equations can model the formation of a spherical black hole by gravitational collapse or describe the evolution of galaxies and globular clusters. We present high-order numerical schemes based on semi-Lagrangian techniques. The convergence of the solution of the discretized problem to the exact solution is proven and high-order error estimates are supplied. More precisely the metric coefficients converge in $L^{\infty}$ and the statistical distribution function of the matter and its moments converge in $L^{2}$ with a rate of $\mathcal{O}\left(\Delta t^{2}+h^{m} / \Delta t\right)$, when the exact solution belongs to $H^{m}$.
\end{abstract}

Mathematics Subject Classification. 65M15, 65P40, 83C05.

Received January 14, 2009. Revised September 7, 2009.

Published online February 4, 2010.

\section{INTRODUCTION}

One of the key outstanding issues in classical general relativity is the determination of the nature of the singularities that result from gravitational collapse. We consider here the Vlasov-Einstein system, which describes self-gravitational collisionless matter. In astrophysics this system models the evolution of galaxies and globular clusters.

Numerical simulation in general relativity is a challenging approach for finding solutions of the Vlasov-Einstein system that describe realistic physical phenomena such as the critical collapse where there are no rigorous analytical results, provided that the numerical scheme we use gives the right solution. The Lagrangian methods called PIC (Particle-In-Cell) methods and its alternatives are the most popular in plasma physics and in the astrophysic communauty [13-15,24,25,27-29]. In [24], the authors studied numerically the critical collapse for the Vlasov-Einstein system in Schwarzschild coordinates in the setting of spherically symmetric asymptotically flat case by means of a PIC method. Rein and Rodewis in [22] proved the convergence of this numerical scheme. In this paper we study a semi-Lagrangian scheme for the spherically symmetric Vlasov-Einstein system and

\footnotetext{
Keywords and phrases. Vlasov-Einstein system, semi-Lagrangian methods, convergence analysis, general relativity.

* The authors acknowledge financial support from the HYKE project No HPRN-CT-2002-00282 on "Hyperbolic and Kinetic Equations: Asymptotics, Numerics, Applications", funded by European Union.

${ }^{1}$ Departamento de Matemática Aplicada Facultad de Ciencias, Universidad de Granada, Avda. Fuentenueva s/n, 18071 Granada, Spain. phbe@ugr.es

2 Institut de Mathématiques Elie Cartan \& Institut Jean Lamour, Département Physique de la Matière et des Matériaux, Nancy-Université, Université Henri Poincaré, BP 70239, 54506 Vandœuvre-lès-Nancy Cedex, France. besse@iecn.u-nancy.fr
} 
we shall prove that the approximate solution converges to the exact solution of the continuous counterpart of the discrete problem when the discretization parameters tend to zero. It seems that semi-Lagrangian schemes have never been used in this context. Semi-Lagrangian methods have given a lot of satisfactory results in various fields such as fluids mechanics, plasma physics and atmospheric sciences $[4,9,12,30]$.

There are several reasons to think that semi-Lagrangian methods could present advantages over usual Lagrangian schemes and thus provide a useful tool for the community of people who work on general relativity. Firstly, since the question of critical collapse is quite relevant in general relativity, it would be worthwhile to develop different schemes to really see whether theses new schemes can confirm the previous results or whether they could deliver new effects. Secondly, as it has been pointed out in [14], where Lagrangian PIC methods have been used, the development of codes which solve the Vlasov equation directly in phase-space would be the best way to get more accurate results since convergence and high-order accuracy properties of theses schemes are better controlled and understood. For semi-Lagrangian schemes we can refer to $[5,6,8]$. The simulations based on the well-known Particle-In-Cell (PIC) methods [11,18], have difficulties to supply a usefully precise description of the particles acceleration process in the regions of phase-space where nonlinear resonant wave-particle interaction (where particle and phase velocities are comparable) may impact the properties of the transport. In fact the PIC codes lack of enough particles to display the detailed phase-space structure of the distribution function in these regions. Indeed it is well known [19,20,22,26,31,32] that the numerical noise of PIC methods decreases as $\mathcal{O}(1 / \sqrt{N})$ where $N$ is the number of particles. On the other hand, direct solution of the Vlasov partial differential equation itself on a phase-space grid (the so called Vlasov codes) have been found to be a powerful tool for studying in details the particle dynamics due to the very fine resolution in phase-space (for example $[4,9,10,12])$ which is ensuring at any point of the phase-space-mesh by high-order a priori estimates. Moreover the Vlasov equation is well-known to often lead to the filamentation or phase-space mixing process, which is one of the reasons why Vlasov simulations have been poorly considered, compared to PIC simulations which are not sensitive to this problem. The distribution function is constant along the characteristic curves which tend to roll up, so that the phase-space regions where the distribution function has different values, come close together and steep gradients are thus generated. Furthermore these mechanisms can strongly be amplified by relativistic effects via strong particle acceleration. In [2] it was also pointed out that the evolution of the solution can become more and more peaked at some spatial point and very fine grid should be used to resolve such sharp peaks. As in [2] the authors do not use an adaptive mesh (which changes with time) but a fixed one, and since the solutions get more and more peaked as time goes on, their grid is not sufficiently fine after a certain time of the collapse. As a consequence, errors on preserved quantities increase. One way to resolve this problem and follow the evolution of the solution further in time is to consider adaptive numerical schemes. In fact, in [10] a wavelet-multiresolution-analysis-based adaptive semi-Lagrangian scheme was developed to solve a reduced Relativistic Vlasov-Maxwell (RVM) system. In [10] and references therein, it has been shown that wavelet multiresolution analysis provides a powerful tool to deal with adaptive numerical schemes. Moreover this reduced RVM system presents a lot of similarities with the reduced Vlasov-Einstein system in the spherically symmetric asymptotic flat case. Each system is two dimensional in phase space $\left((r, w)\right.$ for VE and $\left(x, v_{x}\right)$ for RVM) and each distribution function is parameterized by an invariant, the square of the angular momentum for VE and the canonical transverse momentum for RVM. The main difference between the two systems comes from the vector field which generates the Lagrangian flow and the field equations (electromagnetic fields versus metric coefficients). In a next work we plan to adapt the scheme implemented in [10] according to the semiLagrangian scheme described in this paper to obtain an adaptive semi-Lagrangian Vlasov code which can solve the reduced Vlasov-Einstein system in spherically symmetric asymptotic flat case with Schwarschild and/or maximal-areal coordinates.

In the case of the Vlasov-Einstein system the transport is nonlinear in both physical and momentum space. Therefore the splitting method applied to the advective form of the Vlasov equation is useless and we choose to solve the full nonlinear (ODEs) by a Newton algorithm for example. With this approach two new difficulties arise: The first one is that the Lagrangian flow, associated to the Vlasov-Einstein system, is no more incompressible in the phase-space (as it is true for the Vlasov-Poisson or Vlasov-Maxwell system) and thus the control 
of the Jacobian of the flow is crucial. The second one is that we cannot use the methodology developed in $[5,6,8]$ based on the splitting technique to prove the stability of the scheme. Therefore the present paper constitutes a new step in the development of the theory of the convergence of semi-Lagrangian schemes.

In this paper we present a semi-Lagrangian scheme for the Vlasov-Einstein system in Schwarzschild coordinates for the spherically symmetric asymptotically flat case. Even though it might be more valuable to consider maximal-areal coordinates as in $[2,14]$ because the coordinates can penetrate the event-horizon whereas Schwarzchild coordinates cannot, we conjecture that the convergence proof can be done with maximal-areal coordinates as the degree of nonlinearity and regularity for the vector field (which generates the Lagrangian flow) and the metric coefficient equations are quite similar.

The paper is organized as follows. In the first section we give some basic informations about the spherically symmetric Vlasov-Einstein system in the asymptotically flat case. In the second section, we present the numerical approximation of the solution and in the third section we give the convergence theorem. Finally the last section is devoted to the convergence proof and the statement of error estimates.

\section{The Vlasov-Einstein system in Schwarzschild CoOrdinates}

We consider the Vlasov-Einstein system in spherical symmetry in the case of asymptotically flat spacetimes. Using Schwarzschild coordinates, the non-negative statistical distribution function $f$ of a large ensemble of fully relativistic particles satisfies the set of two-dimensional Vlasov equations

$$
\partial_{t} f+\mathrm{e}^{\mu-\lambda} \frac{w}{\gamma} \partial_{r} f+\left(-\dot{\lambda} w-\mathrm{e}^{\mu-\lambda} \mu^{\prime} \gamma+\mathrm{e}^{\mu-\lambda} \frac{\ell}{r^{3} \gamma}\right) \partial_{w} f=0, \quad \forall \ell \in \mathbb{R}_{+}
$$

where the three-dimensional distribution function $f=f(t, r, w, \ell)$ depends on the radial position $r$, the radial velocity $w$ and the square of the angular momentum $\ell$ which is an invariant of the system. The relativistic factor $\gamma$ reads

$$
\gamma=\sqrt{1+w^{2}+\frac{\ell}{r^{2}}}
$$

while the metric coefficients $\lambda=\lambda(t, r)$ and $\mu=\mu(t, r)$ determine the gravitational field. The solution of the Vlasov equation (2.1) is launched by the initial condition

$$
f(0, r, w, \ell)=f_{0}(r, w, \ell)
$$

where $f_{0}$ is a spherically symmetric, non-negative, continuously differentiable and compactly supported function, such that

$$
4 \pi^{2} \int_{\varrho \leq r} \int_{\mathbb{R}_{\mathbb{R}_{+}}} \gamma f_{0}(\varrho, w, \ell) \mathrm{d} \varrho \mathrm{d} w \mathrm{~d} \ell<\frac{r}{2}, \quad r \geq 0 .
$$

This last condition will be justified further. The assumption on asymptotic flatness is expressed as

$$
\lim _{r \rightarrow \infty} \lambda(t, r)=\lim _{r \rightarrow \infty} \mu(t, r)=0, \quad t \geq 0 .
$$

Moreover we suppose that

$$
\lambda(t, 0)=0, \quad t \geq 0 .
$$

Using boundary conditions (2.3)-(2.4), the Einstein equations can be solved explicitly for the metric coefficients $\mu$ and $\lambda$ and their derivatives. Therefore the integrated Einstein equations read

$$
\mathrm{e}^{-2 \lambda(t, r)}=1-\frac{2 m(t, r)}{r}
$$


and

where

$$
\mu(t, r)=-\int_{r}^{\infty} \mu^{\prime}(t, s) \mathrm{d} s
$$

$$
\mu^{\prime}(t, r)=\mathrm{e}^{2 \lambda(t, r)}\left(\frac{m(t, r)}{r^{2}}+4 \pi r p(t, r)\right)
$$

and

$$
m(t, r)=4 \pi \int_{0}^{r} s^{2} \rho(t, s) \mathrm{d} s .
$$

In equation (2.7) we denote by $\mu^{\prime}$ the partial derivative with respect to the space variable $r$. The mass-energy density $\rho$ and the density of the radial pressure $p$ are defined by

$$
\begin{aligned}
& \rho(t, r)=\frac{\pi}{r^{2}} \int_{-\infty}^{\infty} \int_{0}^{\infty} \gamma f(t, r, w, \ell) \mathrm{d} \ell \mathrm{d} w \\
& p(t, r)=\frac{\pi}{r^{2}} \int_{-\infty}^{\infty} \int_{0}^{\infty} w^{2} f(t, r, w, \ell) \frac{\mathrm{d} \ell \mathrm{d} w}{\gamma} .
\end{aligned}
$$

Redundant but useful expressions to obtain a priori estimates on partial derivatives of $\lambda$ are

$$
\lambda^{\prime}(t, r)=\mathrm{e}^{2 \lambda(t, r)}\left(-\frac{m(t, r)}{r^{2}}+4 \pi r \rho(t, r)\right),
$$

and

$$
\dot{\lambda}=-4 \pi r \mathrm{e}^{\lambda+\mu} j
$$

where the radial mass current density is defined by

$$
j(t, r)=\frac{\pi}{r^{2}} \int_{-\infty}^{\infty} \int_{0}^{\infty} w f(t, r, w, \ell) \mathrm{d} \ell \mathrm{d} w,
$$

and where $\dot{\lambda}$ denotes the partial derivative of $\lambda$ with respect to the time variable $t$. Note that the assumption (2.2) guarantees the positivity of the right hand side of equation (2.5) and that the ADM mass $m(t, \infty)$ is conserved. In other words it means that no trapped surfaces are present initially. More details on the derivation of this system can be found in [21].

Using the notation $z=(r, w, \ell)$, we define by $Z(s ; t, z)=(R, W, L)(s ; t, r, w, \ell)$ the solution of the following differential equations of first order

$$
\begin{aligned}
\frac{\mathrm{d} R}{\mathrm{~d} s}(s)=F_{r}(s, Z(s))= & \mathrm{e}^{(\mu-\lambda)(s, R(s))} \frac{W(s)}{\gamma(Z(s))} \\
\frac{\mathrm{d} W}{\mathrm{~d} s}(s)=F_{w}(s, Z(s))= & -\dot{\lambda}(s, R(s)) W(s)-\mathrm{e}^{(\mu-\lambda)(s, R(s))} \mu^{\prime}(s, R(s)) \gamma(Z(s)) \\
& +\mathrm{e}^{(\mu-\lambda)(s, R(s))} \frac{L(s)}{R^{3}(s) \gamma(Z(s))}, \\
\frac{\mathrm{d} L}{\mathrm{~d} s}(s)=F_{\ell}(s, Z(s))= & 0,
\end{aligned}
$$

with the initial condition $Z(t ; t, z)=z$ and $z=(r, w, \ell) \in Q=\mathbb{R}_{+} \times \mathbb{R} \times \mathbb{R}_{+}$. Let us set $F=\left(F_{r}, F_{w}, F_{\ell}\right)$, the vector field which generates the characteristic flow $Z(s ; t, z)$. Then the system $(2.14)-(2.16)$ can be written in compact form as

$$
\frac{\mathrm{d} Z}{\mathrm{~d} s}(s)=F(s, Z(s))
$$


Therefore the Vlasov equation (2.1), which expresses the fact that the distribution function $f$ remains constant along the integral curves of the vector field $F$ is equivalent to

$$
f(t, r, w, \ell)=f(s,(R, W, L)(s ; t, r, w, \ell))=f(s, Z(s ; t, z)) .
$$

Note that the quantity $L$ is conserved along the characteristics so that the characteristic system (2.14)-(2.16) is essentially two dimensional. While distribution function $f$ is constant along the characteristic curves, the characteristic flow is not volume preserving. More exactly we have:

Lemma 2.1. Let $Z(\cdot ; t, z)=(R, W, L)(\cdot ; t, r, w, \ell)$ denote the solution of the characteristic system associated to (2.1) with $Z(t ; t, z)=z \in Q=\mathbb{R}_{+} \times \mathbb{R} \times \mathbb{R}_{+}$. Then

$$
\operatorname{det} \frac{\partial Z}{\partial z}(s ; t, z)=\mathrm{e}^{\lambda(t, r)-\lambda(s, R(s ; t, z))} .
$$

As an immediate consequence the following quantity

$$
4 \pi^{2} \int \mathrm{e}^{\lambda(t, r)} f(t, r, w, \ell) \mathrm{d} r \mathrm{~d} w \mathrm{~d} \ell
$$

related to the number of particles is conserved. Let us now give a local in time existence result and some useful a priori estimates for the spherically symmetric Vlasov-Einstein system in the case of asymptotically flat spacetimes.

Theorem 2.1. Let us suppose that $f_{0}$ is a non-negative, spherically symmetric, compactly supported function in $\mathscr{C}^{1}(Q) \cap H^{m}(Q)$, which satisfies (2.2), then there exists a unique, regular, spherically symmetric, compactly supported function $f \in \mathscr{C}^{1}([0, T] \times Q) \cap H_{\mathrm{loc}}^{m}([0, T] \times Q)$ which satisfies the Vlasov-Einstein system $(2.1)$ on $[0, T]$ with $f(0, r, w, \ell)=f_{0}(r, w, \ell)$. Moreover we have $\rho, p, j \in \mathscr{C}^{1}\left([0, T] \times \mathbb{R}_{+}\right)$and $m, \lambda, \mu \in \mathscr{C}^{2}\left([0, T] \times \mathbb{R}_{+}\right)$as functions of $t$ and $r$.

Proof. We refer to [21,22] for the proof in continuously differentiable functions space and to [16] for the proof in Sobolev spaces.

In fact, Theorem 2.1 is also true if we suppose that

$$
4 \pi^{2} \int_{\varrho \leq r} \int_{\mathbb{R}^{\prime} \mathbb{R}_{+}} \gamma f_{0}(\varrho, w, \ell) \mathrm{d} \varrho \mathrm{d} w \mathrm{~d} \ell<\frac{r}{2 I}, \quad r \geq 0, \quad I>1,
$$

instead of (2.2). Moreover we have the following lemma.

Lemma 2.2. There exists $I>1$ such that

$$
4 \pi^{2} \int_{\varrho \leq r} \int_{\mathbb{R}^{\prime} \mathbb{R}_{+}} \gamma f(t, \varrho, w, \ell) \mathrm{d} \varrho \mathrm{d} w \mathrm{~d} \ell<\frac{r}{2 I}, \quad r \geq 0, \quad \forall t \in[0, T] .
$$

Proof. We have

$$
\begin{aligned}
m(t, r) & =4 \pi^{2} \int_{\varrho \leq r} \int_{\mathbb{R}_{\times} \mathbb{R}_{+}} \gamma f(t, \varrho, w, \ell) \mathrm{d} \varrho \mathrm{d} w \mathrm{~d} \ell=4 \pi \int_{0}^{r} s^{2} \rho(t, s) \mathrm{d} s \\
& \leq m(t, \infty)=H .
\end{aligned}
$$

The last term is the ADM mass which is an invariant quantity of the system, independent of time $t$. Let $R>0$ be such that $R>4 m(t, \infty)=4 H$, then the function $\lambda(t, r) \in \mathscr{C}^{2}([0, T] \times[0, R])$ is bounded and there exists a constant $M>0$ such that $0 \leq|\lambda(t, r)|<M, \forall t \in[0, T], \forall r \leq R$. Therefore the expression $m(t, r)=r\left(1-\mathrm{e}^{-2 \lambda(t, r)}\right) / 2$ implies $0 \leq m(t, r)<r\left(1-\mathrm{e}^{-2 M}\right) / 2$. Now let us choose $I=\min \left\{2,\left(1-\mathrm{e}^{-2 M}\right)^{-1}\right\}$. Then we get $0 \leq m(t, r)<r /(2 I), \forall t \in[0, T], \forall r \in \mathbb{R}^{+}$. 
Remark 2.1. It is proven in [21] that for appropriate initial datum the Vlasov-Einstein system launches a unique smooth solution in $\mathscr{C}^{1}$. This solution can be extended in time as long as the density $\rho$ or the momentum support is bounded. A smallness assumption on the initial data allows to get a global geodesically complete solution which decays to flat Minkowski space when time goes to infinity [21]. In [23] it is proven that if a solution of the spherically symmetric Vlasov-Einstein system develops a singularity then the first singularity has to appear at the center of the symmetry. Such a break-down of the solution in a particular system of coordinates may not have anything to do with a true spacetime singularity where some geometric quantity or curvature invariants such that the Kretschmann scalar $R_{\alpha \beta \gamma \delta} R^{\alpha \beta \gamma \delta}$ ( $R_{\alpha \beta \gamma \delta}$ is the Riemann curvature tensor) should blow up. However the general picture is that Schwarzschild coordinates are singularity avoiding and the standard but still unproven conjecture is that the global existence holds in these coordinates.

Let us now consider the Vlasov-Einstein system $(2.1)$ in variables $(r, w, \ell)$, with an additional assumption on the support of the initial data described as follows:

$$
\operatorname{supp} f_{0} \subset\left[r_{\min }, r_{\max }\right] \times\left[w_{\min }, w_{\max }\right] \times\left[\ell_{\min }, \ell_{\max }\right], \quad r_{\min }>0, \quad \ell_{\min }>0
$$

Let $T>0$ be fixed such that Theorem 2.1 is verified, we then have following a priori estimates.

Lemma 2.3. There exists a constant $K \geq 1$ such that for all $t \in[0, T]$ and $r \geq 0$,

$$
\rho(t, r), p(t, r),|j(t, r)|, \mathrm{e}^{2 \lambda(t, r)},|\dot{\lambda}(t, r)|,\left|\lambda^{\prime}(t, r)\right|,\left|\lambda^{\prime \prime}(t, r)\right|,\left|\dot{\lambda}^{\prime}(t, r)\right|,|\mu(t, r)|,\left|\mu^{\prime}(t, r)\right|,\left|\mu^{\prime \prime}(t, r)\right| \leq K,
$$

and for $t \in[0, T]$ and $(r, w, \ell) \in \operatorname{supp} f_{0}$,

$$
R(t ; 0, r, w, \ell), \frac{1}{R(t ; 0, r, w, \ell)},|W(t ; 0, r, w, \ell)| \leq K .
$$

Proof. Let see [22] for the proof.

\section{Numerical APPROXimation}

In this part we describe the numerical scheme.

\subsection{Numerical scheme}

Let $\Omega$ (resp. $\Omega_{r}$ ) be the domain of the phase-space (resp. the radial component of the total phase-space $\Omega$ ) on which we compute the numerical solution and $\mathcal{M}_{h}$ a discretization of $\Omega$ where $h$ denotes the space-phase discretization parameter, in other words the maximum size of the cells of the mesh. Let us introduce the Sobolev spaces $H^{m}$ equipped with the following norm

$$
\|f\|_{H^{m}}=\left(\int_{\mathbb{R}_{\xi}^{3}}\left(1+|\xi|^{2}\right)^{m}|\widehat{f}(\xi)|^{2} \mathrm{~d} \xi\right)^{1 / 2}
$$

where $\widehat{f}$ is the Fourier transform of $f$.

In order to construct our algorithm, we need to introduce an approximation operator $\Pi_{h}$ defined on the Sobolev space $H^{m}, m \in \mathbb{N}$. Let us consider a sequence of finite dimensional spaces $V_{h} \subset H^{m}$ such that $\overline{\bigcup_{h \geq 0} V_{h}}=H^{m}$. If the sequence $\left\{\Psi_{k}\right\}$ is a basis of $V_{h}$, we then have

$$
\begin{aligned}
& \Pi_{h}: H^{m}(\Omega) \longrightarrow V_{h} \\
& f \quad \longrightarrow \quad \Pi_{h} f=\sum_{k} c_{k} \Psi_{k} \text {. }
\end{aligned}
$$

In addition we make the following assumptions on $\Pi_{h}$. 
Assumptions 3.1. Let $g \in H^{m}(\Omega)$, and $p, k, m \in \mathbb{N}$ with $k \leq m$ and $\alpha \in[0,1]$, then

(i) Regularity:

$$
\Pi_{h} g \in \mathscr{C}^{p, \alpha}(\Omega) \cap H^{k}(\Omega)
$$

(ii) Consistency and accuracy:

$$
\left\|\Pi_{h} g-g\right\|_{H^{k}(\Omega)} \leq c h^{m-k}\|g\|_{H^{m}(\Omega)} .
$$

(iii) Stability:

$$
\left\|\Pi_{h} g\right\|_{H^{k}(\Omega)} \leq\|g\|_{H^{k}(\Omega)} .
$$

Moreover we use the following property [17]: there exists a constant $K_{1}>0$ such that

$$
\forall v_{h} \in V_{h}, \quad\left\|v_{h}\right\|_{H^{m+1}(\Omega)} \leq K_{1} h^{-1}\left\|v_{h}\right\|_{H^{m}(\Omega)} .
$$

This last inequality is called an inverse inequality. Such reconstruction operator $\Pi_{h}$ exists and we can refer to [3], for an example in the context of wavelets multiresolution analysis. In fact in [3], it is shown that we can construct a multiresolution analysis in $H^{s}$ where the orthonormal wavelets basis is compactly supported and have the desired smoothness, and such that the orthogonal projection operator can reproduce all polynomials less or equal to any fixed order, i.e. has the property of high-order approximation or accuracy. Since our scheme could use wavelet reconstruction, it can be modified to get an adaptive scheme by adding a step of prediction (which will consist in predicting the new mesh where the solution will be computed by pushing the characteristic curves forward) before solving the characteristics backward and a step of wavelets decomposition and data compression after this latter. This procedure which has been implemented in [10] to obtain a wavelet-multiresolution-analysisbased adaptive semi-Lagrangian scheme for solving a reduced Relativistic Vlasov-Maxwell system, reveals to be a useful and powerful tool to deal with steep gradients (which generate small scales) and moving sharp peaks which occur during the filamentation mechanism and the phase-space mixing process.

In the next section we present a first-order in time scheme and a second-order in time scheme. If the time $T$ is the final evolution time of Theorem 2.1 and $N_{T} \in \mathbb{N}^{*}$ the number of time iterations, we then define the time step $\Delta t=T / N_{T}$ and the time instant $t^{n}=n \Delta t$. Let us suppose that we know $f_{h}^{n} \in V_{h}$, an approximation of the distribution function $f$ on a regular Cartesian mesh $\mathcal{M}_{h}$ of the $(r, w, \ell)$-phase-space at time $t=t^{n}$, the numerical scheme then describes the way to construct $f_{h}^{n+1}$ from the known data $f_{h}^{n}$.

\subsubsection{The first-order scheme in time}

Using the fact that $f$ is constant along the characteristic curves (2.18) we define

$$
f_{h}^{n+1}(r, w, \ell):=\Pi_{h} f_{h}^{n}\left(R_{h}\left(t^{n}\right), W_{h}\left(t^{n}\right), L_{h}\left(t^{n}\right)\right),
$$

where $Z_{h}\left(t^{n}\right)=\left(R_{h}\left(t^{n}\right), W_{h}\left(t^{n}\right), L_{h}\left(t^{n}\right)\right)$ is an approximation of the solution of the system (2.14)-(2.16) with the final condition $Z_{h}\left(t^{n+1}\right)=\left(R_{h}\left(t^{n+1}\right), W_{h}\left(t^{n+1}\right), L_{h}\left(t^{n+1}\right)\right)=(r, w, \ell)=z \in \mathcal{M}_{h}$.

In the case of the first-order time discretization, with the notation $Z_{h}^{n}=Z_{h}\left(t^{n}\right)$, we define

$$
Z_{h}^{n+1}-Z_{h}^{n}=\Delta t F_{h}^{n}\left(Z_{h}^{n}\right),
$$

where $F_{h}^{n}=\left(F_{h, r}^{n}, F_{h, w}^{n}, F_{h, \ell}^{n}\right)$ are obtained by replacing in $F(t,$.$) the quantities \lambda(t), \mu(t), \mu^{\prime}(t)$ and $\dot{\lambda}(t)$ at time $t=t^{n}$ by their approximations $\lambda_{h}^{n}, \mu_{h}^{n}, \mu_{h}^{n \prime}$ and $\dot{\lambda}_{h}^{n}$. The metric coefficients $\lambda_{h}^{n}, \mu_{h}^{n}, \mu_{h}^{n \prime}, \dot{\lambda}_{h}^{n}$ and the mass $m_{h}^{n}$ are constructed by replacing in equations (2.12) and (2.5)-(2.8) the moments $\rho(t), p(t), j(t)$ by their approximations $\rho_{h}^{n}, p_{h}^{n}, j_{h}^{n}$, where the latter are computed by replacing in the definition of the moments (2.9)(2.10) and (2.13) $f(t)$ by $f_{h}^{n}$. 


\section{Remark 3.1.}

(1) Note that $\left(\lambda^{\prime}\right)_{h}=\lambda_{h}^{\prime}$ and $\left(\dot{\lambda_{h}}\right) \neq(\dot{\lambda})_{h}$.

(2) Equation (3.6) is a first-order discretization in time (Euler scheme) of the system (2.17).

(3) In fact equation (3.6) is a fixed point problem in the unknown $Z_{h}^{n}$. In order to solve this fixed point we can use a Newton method. A sufficient condition to have local convergence of the Newton algorithm is that $F_{h}^{n} \in \mathscr{C}^{1,1}(\Omega)$ and that $\nabla F_{h}^{n}$ is locally invertible or that the Jacobian of $F_{h}^{n}$ does not vanish on a neighbourhood of the solution. In our case we shall show that these conditions are fulfilled by $F_{h}^{n}$.

\subsubsection{The second-order scheme in time}

Here we present the second-order scheme in time which is composed by two successive first-order schemes in time.

(1) If we suppose that we know $f_{h}^{n} \in V_{h}$ we then set

$$
f_{h}^{n+1 / 2}(r, w, \ell):=\Pi_{h} f_{h}^{n}\left(\widehat{R}_{h}\left(t^{n}\right), \widehat{W}_{h}\left(t^{n}\right), \widehat{L}_{h}\left(t^{n}\right)\right),
$$

where $\widehat{Z}_{h}\left(t^{n}\right)=\left(\widehat{R}_{h}\left(t^{n}\right), \widehat{W}_{h}\left(t^{n}\right), \widehat{L}_{h}\left(t^{n}\right)\right)$ is the approximation of the solution of the system $(2.14)-(2.16)$ with the final condition $\widehat{Z}_{h}\left(t^{n+1 / 2}\right)=\left(\widehat{R}_{h}\left(t^{n+1 / 2}\right), \widehat{W}_{h}\left(t^{n+1 / 2}\right), \widehat{L}_{h}\left(t^{n+1 / 2}\right)\right)=(r, w, \ell)=z \in \mathcal{M}_{h}$. The unknown $\widehat{Z}_{h}\left(t^{n}\right)$ is computed using the equation

$$
\widehat{Z}_{h}^{n+1 / 2}-\widehat{Z}_{h}^{n}=\frac{\Delta t}{2} F_{h}^{n}\left(\widehat{Z}_{h}^{n}\right) .
$$

(2) From the distribution function $f_{h}^{n+1 / 2}$ and equations (2.9)-(2.10) and (2.13), we get the moments $\rho_{h}^{n+1 / 2}, p_{h}^{n+1 / 2}, j_{h}^{n+1 / 2}$. Using equations (2.12) and (2.5)-(2.8) we obtain $\lambda_{h}^{n+1 / 2}, \mu_{h}^{n+1 / 2}, \mu_{h}^{n+1 / 2}$, $\dot{\lambda}_{h}^{n+1 / 2}$ and consequently we get $F_{h}^{n+1 / 2}$.

(3) We then set

$$
f_{h}^{n+1}(r, w, \ell):=\Pi_{h} f_{h}^{n}\left(R_{h}\left(t^{n}\right), W_{h}\left(t^{n}\right), L_{h}\left(t^{n}\right)\right),
$$

where $Z_{h}\left(t^{n}\right)=\left(R_{h}\left(t^{n}\right), W_{h}\left(t^{n}\right), L_{h}\left(t^{n}\right)\right)$ is the approximation of the solution of the system (2.14)(2.16) with the final condition $Z_{h}\left(t^{n+1}\right)=\left(R_{h}\left(t^{n+1}\right), W_{h}\left(t^{n+1}\right), L_{h}\left(t^{n+1}\right)\right)=(r, w, \ell)=z \in \mathcal{M}_{h}$. The new unknown $Z_{h}\left(t^{n}\right)$ is approximated by the expression

$$
Z_{h}^{n+1}-Z_{h}^{n}=\Delta t F_{h}^{n+1 / 2}\left(Z_{h}^{n+1 / 2}\right),
$$

with the definition $Z_{h}^{n+1 / 2}=\left(Z_{h}^{n+1}+Z_{h}^{n}\right) / 2$.

The fixed point problem (3.8) and (3.10) can also be solved by a Newton algorithm. We shall also show that the conditions on $F_{h}^{n}$ and $F_{h}^{n+1 / 2}$ are fulfilled.

Remark 3.2. Let us note that a similar second-order in time Runge-Kutta semi-Lagrangian scheme with B-spline interpolation has been successfully implemented to solve a three-dimensional multi-fluid model for gyrokinetic turbulence in [7]. Moreover following the derivation of the second-order scheme in time of Section 3.1.2 it is formally possible to write down higher-order schemes in time (with order greater than two) by considering Runge-Kutta schemes with more than two stages. Obviously such schemes become highly intricate and thus are more complicated to analyze. 


\section{Convergence theorem And a Priori ERror EStimates}

In this section we give the convergence theorem for the schemes presented in Section 3.1.

Theorem 4.1. Let us assume that $f_{0} \in \mathscr{C}^{s+1}(\Omega) \cap H^{m}(\Omega)$ and fulfills the condition (2.19) of Theorem 2.1, and there exist two constants $C_{1}$ and $C_{2}$ such that $C_{1} \leq h^{-1} \Delta t \leq C_{2}$. Then the approximation $\left(f_{h}, \lambda_{h}, \mu_{h}\right)$ of the Vlasov-Einstein system presented in Section 3.1 converges towards the solution of Theorem 2.1 on the time interval $[0, T]$ and for $k \leq 1$ we have the following error estimates

$$
\begin{aligned}
& \sup _{n \leq N_{T}}\left\|f_{h}^{n}-f\left(t^{n}\right)\right\|_{H^{k}(\Omega)} \lesssim \Delta t^{s}+\frac{h^{m-k}}{\Delta t} \\
& \sup _{n \leq N_{T}}\left\{\left\|\lambda_{h}^{n}-\lambda\left(t^{n}\right)\right\|_{L^{\infty}\left(\Omega_{r}\right)},\left\|\mu_{h}^{n}-\mu\left(t^{n}\right)\right\|_{L^{\infty}\left(\Omega_{r}\right)},\left\|m_{h}^{n}-m\left(t^{n}\right)\right\|_{L^{\infty}\left(\Omega_{r}\right)}\right\} \lesssim \Delta t^{s}+\frac{h^{m}}{\Delta t} \\
& \sup _{n \leq N_{T}}\left\{\left\|\rho_{h}^{n}-\rho\left(t^{n}\right)\right\|_{H^{k}\left(\Omega_{r}\right)},\left\|p_{h}^{n}-p\left(t^{n}\right)\right\|_{H^{k}\left(\Omega_{r}\right)},\left\|j_{h}^{n}-j\left(t^{n}\right)\right\|_{H^{k}\left(\Omega_{r}\right)}\right\} \lesssim \Delta t^{s}+\frac{h^{m-k}}{\Delta t}
\end{aligned}
$$

with $s=1$ for the first-order scheme of Section 3.1.1 and $s=2$ for the second-order scheme of Section 3.1.2.

Remark 4.1. The assumption $f_{0} \in \mathscr{C}^{s+1}(\Omega)$ with $s=1$ (resp. $s=2$ ) in Theorem 4.1, is just a technical assumption which is used to bound the terms $\left\|\partial_{t} F\right\|_{L^{\infty}\left(0, T ; H^{k}(\Omega)\right)}\left(\right.$ resp. $\left\|\partial_{t}^{2} F\right\|_{L^{\infty}\left(0, T ; H^{k}(\Omega)\right)}$ and $\left.\left\|\partial_{t} \nabla F\right\|_{L^{\infty}\left(0, T ; H^{k}(\Omega)\right)}\right)$, with $k \leq 1$, in the proof of the first (resp. second) order scheme.

\section{Convergence Analysis}

Let $T_{h}<T$ denote the maximal time on which the approximate solution exists and satisfies the a priori estimates

$$
2 m_{h}^{n}(r)<r ; \quad\left|\lambda_{h}^{n}\right|,\left|\lambda_{h}^{\prime n}\right|,\left|\dot{\lambda}_{h}^{n}\right|,\left|\mu_{h}^{n}\right|,\left|\mu_{h}^{\prime n}\right| \leq 2 K, \quad n \leq N_{T_{h}},
$$

where $K$ is the constant used in Lemma 2.3. Note that we have $T_{h}>0$ by continuity in time. Using a reductio ad absurdum argument, we shall prove that in fact $T_{h}=T$ at the end of Section 5.1. We now decompose the error $E^{n+1}$ at time $t^{n+1}$ in three parts as it follows

$$
\begin{aligned}
E^{n+1}= & \left\|f\left(t^{n+1}\right)-f_{h}^{n+1}\right\|_{X(\Omega)} \\
\leq & \left\|f^{n}\left(R^{n}, W^{n}, L^{n}\right)-\Pi_{h} f^{n}\left(R^{n}, W^{n}, L^{n}\right)\right\|_{X(\Omega)} \\
& +\left\|\Pi_{h}\left(f^{n}\left(R^{n}, W^{n}, L^{n}\right)-f^{n}\left(R_{h}^{n}, W_{h}^{n}, L_{h}^{n}\right)\right)\right\|_{X(\Omega)} \\
& +\left\|\Pi_{h}\left(f^{n}\left(R_{h}^{n}, W_{h}^{n}, L_{h}^{n}\right)-f_{h}^{n}\left(R_{h}^{n}, W_{h}^{n}, L_{h}^{n}\right)\right)\right\|_{X(\Omega)} \\
= & E 1+E 2+E 3 .
\end{aligned}
$$

In the above the $X$-norm will denote some Sobolev $H^{m}$-norm.

Let us compute first the interpolation error $E 1$ which depends only on the approximation and accuracy property (3.2) of the reconstruction operator $\Pi_{h}$. From (3.2) we get for $k=0,1$,

$$
\begin{aligned}
E 1 & =\left\|f^{n}\left(R^{n}, W^{n}, L^{n}\right)-\Pi_{h} f^{n}\left(R^{n}, W^{n}, L^{n}\right)\right\|_{H^{k}(\Omega)} \\
& =\left\|f^{n}\left(Z^{n}\right)-\Pi_{h} f^{n}\left(Z^{n}\right)\right\|_{H^{k}(\Omega)} \\
& \lesssim h^{m-k}\left\|f^{n}\left(Z^{n}\right)\right\|_{H^{m}(\Omega)} \\
& \lesssim h^{m-k}\left(\left\|\operatorname{det}^{-1} \partial_{z} Z^{n}\right\|_{L^{\infty}(\Omega)} \max _{|\alpha|, \beta \leq m}\left\|\partial_{z}^{\alpha} Z^{n}\right\|_{L^{\infty}(\Omega)}^{\beta}\right)^{1 / 2}\left\|f^{n}\right\|_{H^{m}(\Omega)} \\
& \lesssim h^{m-k}\left\|f^{n}\right\|_{H^{m}(\Omega)} \lesssim h^{m-k}\|f\|_{L^{\infty}\left([0, T] ; H^{m}(\Omega)\right)} .
\end{aligned}
$$




\subsection{Convergence of the first-order in time scheme}

Let us first give a proposition which states the boundness of the support of the distribution function.

Proposition 5.1. Under the assumptions (5.1), there exists a constant $D$, depending on $K$, but independent of $h$ and $\Delta t$ such that

$$
\frac{1}{R_{h}^{n}}, R_{h}^{n},\left|W_{h}^{n}\right|, L_{h}^{n} \leq D, \quad \forall n \leq N_{T_{h}} .
$$

Proof. As $F_{h, \ell}=0$, then $L_{h}^{n}=L_{h}^{0}=$ constant.

From assumptions (5.1) and the first component of (3.6) we get

$$
\begin{aligned}
R_{h}^{n+1}-R_{h}^{n} & =\Delta t \mathrm{e}^{\mu_{h}^{n}-\lambda_{h}^{n}} \frac{W_{h}^{n}}{\gamma_{h}^{n}} \\
& \leq \Delta t \mathrm{e}^{4 K}
\end{aligned}
$$

Obviously we get $R_{h}^{n} \leq R_{h}^{0}+C T_{h} \leq R_{h}^{0}+C T, n \leq N_{T_{h}}$. Now let us bound $R_{h}^{n}$ from below. Let us suppose first that $W_{h}^{n}<0$. Then using (5.1)

$$
\begin{aligned}
R_{h}^{n+1} & =R_{h}^{n}-\Delta t \mathrm{e}^{\mu_{h}^{n}-\lambda_{h}^{n}} \frac{\left|W_{h}^{n}\right|}{\gamma_{h}^{n}} \\
& \geq R_{h}^{n}-\Delta t \mathrm{e}^{\mu_{h}^{n}-\lambda_{h}^{n}} \\
& \geq R_{h}^{n}-C \Delta t .
\end{aligned}
$$

If $W_{h}^{n}>0$ then obviously $R_{h}^{n+1} \geq R_{h}^{n}$. In both case we have $R_{h}^{n+1} \geq R_{h}^{n}-C \Delta t$ which implies $R_{h}^{n} \geq R_{h}^{0}-C T_{h} \geq$ $R_{h}^{0}-C T$, for all $n \leq N_{T_{h}}$. We have proved that $R_{h}^{n}$ is in the ball of center $R_{0}$ and radius $C T$. We then have to choose $r_{\text {min }} \geq C T$ where $r_{\min }$ is related to the support of $f_{0}$ as in (2.21). The lower bound is not optimal because it corresponds to the bad case, $W_{h}^{n}<0, \forall n \leq N_{T_{h}}$. In fact as the sign of $W_{h}^{n}$ varies with $n$ the lower bounds should be better. Now let us bound $W_{h}^{n}$. Using assumptions (5.1), and the fact that $R_{h}^{n}$ is bounded from below, for the second component of (3.6) we get

$$
\begin{aligned}
W_{h}^{n+1}-W_{h}^{n} & =\Delta t\left(-\dot{\lambda}_{h}^{n} W_{h}^{n}-\mu_{h}^{n \prime} \gamma_{h}^{n} \mathrm{e}^{\mu_{h}^{n}-\lambda_{h}^{n}}+\mathrm{e}^{\mu_{h}^{n}-\lambda_{h}^{n}} \frac{L_{h}^{n}}{\left(R_{h}^{n}\right)^{3} \gamma_{h}^{n}}\right) \\
& \leq C \Delta t\left(1+\left|W_{h}^{n}\right|\right) .
\end{aligned}
$$

The above implies that $\left|W_{h}^{n+1}\right| \leq\left|W_{h}^{0}\right|(1+C \Delta t)^{N_{T_{h}}}+C T_{h} \leq\left|W_{h}^{0}\right| \mathrm{e}^{C T_{h}}+C T_{h} \leq\left|W_{h}^{0}\right| \mathrm{e}^{C T}+C T$, which ends the proof.

Let us now prove the stability of the scheme which allows to control the accumulation error E3.

Lemma 5.1. Under the assumptions (5.1), the scheme is $H^{3}$-stable.

Proof. In fact, in the sequel, we can see that the $H^{2}$-stability is enough to show the convergence of the scheme. In that case the stability property (3.3) with $H^{2}$-norm should be enough. Nevertheless, when we compute the term $\left\|f_{h}^{n+1}\right\|_{H^{2}(\Omega)}$ appears the term $\left|\partial_{w} f_{h}^{n}\right|\left|\partial_{r}^{2} W_{h}^{n}\right|$ which leads us to evaluate the integral

$$
\int_{\Omega}\left|\partial_{w} f_{h}^{n}\right|^{2}\left|\partial_{r}^{2} f_{h}^{n}\right|^{2} \mathrm{~d} r \mathrm{~d} w \mathrm{~d} l
$$

In order get the $H^{2}$-stability we need to have an expression which depends only on the power of $\left\|f_{h}^{n}\right\|_{H^{2}(\Omega)}$. Nevertheless it seems not possible that the term (5.4) could be bounded by a power of $\left\|f_{h}^{n}\right\|_{H^{2}(\Omega)}$. 
Using the continuous Sobolev embedding $H^{2}\left(\mathbb{R}^{3}\right) \hookrightarrow L^{\infty}\left(\mathbb{R}^{3}\right)$ we get the estimate

$$
\int_{\Omega}\left|\partial_{w} f_{h}^{n}\right|^{2}\left|\partial_{r}^{2} f_{h}^{n}\right|^{2} \mathrm{~d} r \mathrm{~d} w \mathrm{~d} l \leq\left\|f_{h}^{n}\right\|_{H^{3}(\Omega)}^{2}\left\|f_{h}^{n}\right\|_{H^{2}(\Omega)}^{2}
$$

which suggests us to deal with the $H^{3}$-stability. In that case, the bad term becomes $\left|\partial_{w} f_{h}^{n}\right|\left|\partial_{r}^{3} W_{h}^{n}\right|$, and leads to the integral term

$$
\int_{\Omega}\left|\partial_{w} f_{h}^{n}\right|^{2}\left|\partial_{r}^{3} f_{h}^{n}\right|^{2} \mathrm{~d} r \mathrm{~d} w \mathrm{~d} l
$$

which is now bounded by a power of $\left\|f_{h}^{n}\right\|_{H^{3}(\Omega)}$. Therefore we should seek the stability of the scheme in $H^{3}$-norm. Now, since the flow is not volume preserving, in order to obtain $H^{3}$-stability, we should control the Jacobian of the approximate flow $Z_{h}$ and its partial derivatives $\partial_{z}^{\alpha} Z_{h}^{n}$ with $|\alpha| \leq 3$. Let us first compute the Jacobian matrix $\partial_{z} Z_{h}^{n}=\left(\nabla_{z} R_{h}^{n}, \nabla_{z} W_{h}^{n}, \nabla_{z} L_{h}^{n}\right)^{T}$. By differentiating (3.6) we have

$$
\begin{aligned}
\partial_{z} Z_{h}^{n} & =\left(I+\Delta t \partial_{z} F_{h}^{n}\left(Z_{h}^{n}\right)\right)^{-1} \\
& =\frac{1}{\mathbb{D}}\left(\begin{array}{ccc}
1+\Delta t \partial_{w} F_{h, w}^{n} & -\Delta t \partial_{w} F_{h, r}^{n} & \Delta t^{2} \partial_{w} F_{h, r}^{n} \partial_{l} F_{h, w}^{n}-\Delta t\left(1+\Delta t \partial_{w} F_{h, w}^{n}\right) \\
-\Delta t \partial_{r} F_{h, w}^{n} & 1+\Delta t \partial_{r} F_{h, r}^{n} & \Delta t^{2} \partial_{l} F_{h, r}^{n} \partial_{r} F_{h, w}^{n}-\Delta t\left(1+\Delta t \partial_{r} F_{h, r}^{n}\right) \\
0 & 0 & \mathbb{D}
\end{array}\right)
\end{aligned}
$$

where $\mathbb{D}$ denotes the Jacobian, equal to

$$
\mathbb{D}=1+\Delta t\left(\partial_{r} F_{h, r}^{n}+\partial_{w} F_{h, w}^{n}\right)+\Delta t^{2}\left(\partial_{r} F_{h, r}^{n} \partial_{w} F_{h, w}^{n}-\partial_{r} F_{h, w}^{n} \partial_{w} F_{h, r}^{n}\right) .
$$

We now need to compute all the second and third derivatives of $Z_{h}^{n}$. In order to bound $\partial_{z}^{\alpha} Z_{h}^{n}$ with $|\alpha| \leq 3$, we need to estimate the approximate force field $F_{h}^{n}$ which generates the approximate flow $Z_{h}^{n}$ as its partial derivatives. Therefore we need the following lemma which estimates the partial derivatives of the force field $F_{h}^{n}$ and whose proof is postponed in Appendix A.

Lemma 5.2. Under the assumptions (5.1) there exists a generic constant $C$, depending on $K$, but independent of $h$ and $\Delta t$ such that for $F_{h, r}^{n}$ and $F_{h, w}^{n}$ we get the following bounds

$$
\begin{aligned}
\left\|\partial_{r}^{m} \partial_{w}^{\alpha} \partial_{l}^{\beta} F_{h, r}^{n}\right\|_{L^{\infty}(\Omega)} & \leq C \begin{cases}1 & \text { if } m \leq 1 \\
\left\|f_{h}^{n}\right\|_{H^{m}(\Omega)} & \text { if } m>1\end{cases} \\
\left\|\partial_{r}^{m} \partial_{w}^{\alpha} \partial_{l}^{\beta} F_{h, w}^{n}\right\|_{L^{\infty}(\Omega)} & \leq C\left\|f_{h}^{n}\right\|_{H^{m+1}(\Omega)} .
\end{aligned}
$$

Remark 5.1. The component $F_{h, w}^{n}$ loses one space derivative with respect to $F_{h, r}^{n}$ since the expression for $F_{h, w}^{n}$ involves $\dot{\lambda}_{h}^{n}$ and $\mu_{h}^{n^{\prime}}$. The derivatives with respect to the variables $w$ and $l$ do not make lose spatial derivatives.

By a straightforward but lengthy calculation, using Lemma 5.2, the computation of $\partial_{z}^{\alpha} Z_{h}^{n}$ for $|\alpha| \leq 3$, the inverse inequality (3.4) and assuming that the ratio $\Delta t / h$ is bounded, we get the following proposition

Proposition 5.2. Under the assumptions (5.1), there exists a generic constant $C$, depending on $K$, but independent of $h$ and $\Delta t$ such that

$$
\begin{aligned}
\|\mathbb{D}\|_{L^{\infty}(\Omega)} & \leq 1+C \Delta t\left\|f_{h}^{n}\right\|_{H^{1}(\Omega)} \\
\left\|\partial_{z}^{\alpha} \mathbb{D}\right\|_{L^{\infty}(\Omega)} & \leq\left(1+\Delta t\left\|f_{h}^{n}\right\|_{H^{2}(\Omega)}\right) C \Delta t\left\|f_{h}^{n}\right\|_{H^{3}(\Omega)}, \quad 1 \leq|\alpha| \leq 2, \\
\left\|\partial_{z_{i}}^{\alpha} Z_{h j}^{n}\right\|_{L^{\infty}(\Omega)} & \leq \delta_{i j}+\left(1+\Delta t\left\|f_{h}^{n}\right\|_{H^{1}(\Omega)}\right) C \Delta t\left\|f_{h}^{n}\right\|_{H^{2}(\Omega)}, \quad|\alpha|=1, \\
\|Y\|_{L^{\infty}(\Omega)} & \leq\left(1+\Delta t\left\|f_{h}^{n}\right\|_{H^{3}(\Omega)}\right) C \Delta t\left\|f_{h}^{n}\right\|_{H^{3}(\Omega)} \quad \forall Y \in\left\{\partial_{z}^{\alpha} Z_{h}^{n}|2 \leq| \alpha \mid \leq 3\right\} \backslash\left\{\partial_{r}^{3} W_{h}^{n}\right\} \\
\left\|\partial_{r}^{3} W_{h}^{n}\right\|_{L^{2}(\Omega)} & \leq\left(1+\Delta t\left\|f_{h}^{n}\right\|_{H^{3}(\Omega)}\right) C \Delta t\left\|f_{h}^{n}\right\|_{H^{3}(\Omega)} .
\end{aligned}
$$


Now we are ready to compute the $H^{3}$-norm of $f_{h}^{n+1}$. Note that with our definition, the $H^{3}$-norm of a function $g$ is given by

$$
\begin{aligned}
\|g\|_{H^{3}(\Omega)}^{2} & =\|g\|_{H^{2}(\Omega)}^{2}+\|\nabla g\|_{H^{2}(\Omega)}^{2} \\
& =\|g\|_{L^{2}(\Omega)}^{2}+3\|\nabla g\|_{L^{2}(\Omega)}^{2}+3\|\Delta g\|_{L^{2}(\Omega)}^{2}+\|\nabla \Delta g\|_{L^{2}(\Omega)}^{2} .
\end{aligned}
$$

If we compute $\nabla f_{h}^{n}\left(Z_{h}^{n}\right), \Delta f_{h}^{n}\left(Z_{h}^{n}\right), \nabla \Delta f_{h}^{n}\left(Z_{h}^{n}\right)$, use the bounds of Proposition 5.2 and the inverse inequality (3.4) and if we assume that the ratio $\Delta t / h$ is bounded, we obtain the following bounds

$$
\begin{aligned}
& \left\|f_{h}^{n}\left(Z_{h}^{n}\right)\right\|_{L^{2}(\Omega)} \leq\|\mathbb{D}\|_{L^{\infty}(\Omega)}\left\|f_{h}^{n}\right\|_{L^{2}(\Omega)} \\
& \leq\left\|f_{h}^{n}\right\|_{L^{2}(\Omega)}+C \Delta t\left\|f_{h}^{n}\right\|_{L^{2}(\Omega)}\left\|f_{h}^{n}\right\|_{H^{1}(\Omega)} \\
& \left\|\nabla\left(f_{h}^{n}\left(Z_{h}^{n}\right)\right)\right\|_{L^{2}(\Omega)} \leq\|\mathbb{D}\|_{L^{\infty}(\Omega)}\left\|\nabla f_{h}^{n}\right\|_{L^{2}(\Omega)}\left\|\nabla Z_{h}^{n}\right\|_{L^{\infty}(\Omega)} \\
& \leq\left\|\nabla f_{h}^{n}\right\|_{L^{2}(\Omega)}+C \Delta t\left\|f_{h}^{n}\right\|_{H^{2}(\Omega)}\left\|f_{h}^{n}\right\|_{H^{1}(\Omega)}\left(1+\Delta t\left\|f_{h}^{n}\right\|_{H^{1}(\Omega)}\right) ; \\
& \left\|\Delta\left(f_{h}^{n}\left(Z_{h}^{n}\right)\right)\right\|_{L^{2}(\Omega)} \leq\left\|\nabla^{2} f_{h}^{n}\left(Z_{h}^{n}\right)\left(\nabla Z_{h}^{n}\right)^{2}+\nabla f_{h}^{n}\left(Z_{h}^{n}\right) \nabla^{2} Z_{h}^{n}\right\|_{L^{2}(\Omega)} \\
& \leq\left\|\Delta f_{h}^{n}\right\|_{L^{2}(\Omega)}+C \Delta t\left\|f_{h}^{n}\right\|_{H^{3}(\Omega)}^{3}\left(1+\Delta t\left\|f_{h}^{n}\right\|_{H^{3}(\Omega)}\right) ; \\
& \left\|\nabla \Delta\left(f_{h}^{n}\left(Z_{h}^{n}\right)\right)\right\|_{L^{2}(\Omega)} \leq\left\|\nabla^{3} f_{h}^{n}\left(Z_{h}^{n}\right)\left(\nabla Z_{h}^{n}\right)^{3}+2 \nabla^{2} f_{h}^{n}\left(Z_{h}^{n}\right) \nabla Z_{h}^{n} \nabla^{2} Z_{h}^{n}+\nabla f_{h}^{n}\left(Z_{h}^{n}\right) \nabla^{3} Z_{h}^{n}\right\|_{L^{2}(\Omega)} \\
& \leq\left\|\nabla \Delta f_{h}^{n}\right\|_{L^{2}(\Omega)}+C \Delta t\left\|f_{h}^{n}\right\|_{H^{3}(\Omega)}^{4}\left(1+\Delta t\left\|f_{h}^{n}\right\|_{H^{3}(\Omega)}\right)+\left\|\partial_{w} f_{h}^{n}\right\|_{L^{\infty}(\Omega)}\left\|\partial_{r}^{3} W_{h}^{n}\right\|_{L^{2}(\Omega)} \\
& \leq\left\|\nabla \Delta f_{h}^{n}\right\|_{L^{2}(\Omega)}+C \Delta t\left\|f_{h}^{n}\right\|_{H^{3}(\Omega)}^{4}\left(1+\Delta t\left\|f_{h}^{n}\right\|_{H^{3}(\Omega)}\right) .
\end{aligned}
$$

If we set $Y^{n+1}=\left\|f_{h}^{n+1}\right\|_{H^{3}(\Omega)}$, inequalities (5.6)-(5.10) then lead to

$$
Y^{n+1} \leq\left(1+C \Delta t\left(Y^{n}\right)^{4}\right) Y^{n} .
$$

If we sum this inequality with respect to $n$, we obtain

$$
Y^{n+1} \leq Y^{0}+C \Delta t \sum_{l=0}^{n} Y^{l^{5}}
$$

Therefore, applying Theorem 4.2.1 [1] with $r=1, p=1, q=Y^{0}$, and $\mathcal{H}_{1}\left(n+1,\left\{Y^{n}\right\}_{n \geq 0}\right)=\sum_{l=0}^{n} C \Delta t Y^{l^{5}}$, yields

$$
Y^{n+1} \leq\left(\frac{1}{Y^{0^{4}}}-4 C T\right)^{-1 / 4}
$$

We have stability only if $T<T_{Y_{0}}=1 /\left(4 C Y^{0^{4}}\right)$. Therefore the scheme is $H^{3}$-stable if we choose $T_{h}$ as the minimum of $T_{Y_{0}}$ and the time $T_{h}$ defined by assumptions (5.1), which completes the proof.

Now let us compute the term $E 3$ which stands for the accumulation error.

Lemma 5.3. Under the assumptions (5.1) we have

$$
E 3 \leq(1+C \Delta t) E^{n} .
$$


Proof. From the stability Lemma 5.1 we have

$$
\left\|\partial_{z} Z_{h}^{n}\right\|_{L^{\infty}(\Omega)},\left\|\operatorname{det}^{-1} \partial_{z} Z_{h}^{n}\right\|_{L^{\infty}(\Omega)} \leq 1+C \Delta t .
$$

From above and using (3.3), we get for $k=0,1$,

$$
\begin{aligned}
E 3 & =\left\|\Pi_{h}\left(f^{n}\left(Z_{h}^{n}\right)-f_{h}^{n}\left(Z_{h}^{n}\right)\right)\right\|_{H^{k}(\Omega)} \\
& \leq\left\|f^{n}\left(Z_{h}^{n}\right)-f_{h}^{n}\left(Z_{h}^{n}\right)\right\|_{H^{k}(\Omega)} \\
& \leq(1+C \Delta t)\left\|f^{n}-f_{h}^{n}\right\|_{H^{k}(\Omega)} \\
& \leq(1+C \Delta t) E^{n},
\end{aligned}
$$

which ends the proof.

Let us now compute the term $E 2$ which stands for time discretization error and coupling error linked to self-consistency.

Lemma 5.4. Under the assumptions (5.1) we have

$$
E 2 \lesssim \Delta t^{2}+\Delta t E^{n}
$$

Proof. For $k=0,1$, we have

$$
\begin{aligned}
E 2 & =\left\|\Pi_{h}\left(f^{n}\left(Z^{n}\right)-f^{n}\left(Z_{h}^{n}\right)\right)\right\|_{H^{k}(\Omega)} \\
& \leq\left\|f^{n}\left(Z^{n}\right)-f^{n}\left(Z_{h}^{n}\right)\right\|_{H^{k}(\Omega)} \\
& \leq\|f\|_{L^{\infty}\left(0, T ; W^{2, \infty}(\Omega)\right)}\left\|Z^{n}-Z_{h}^{n}\right\|_{H^{k}(\Omega)} \\
& \lesssim\left\|Z^{n}-\widetilde{Z}^{n}\right\|_{H^{k}(\Omega)}+\left\|\widetilde{Z}^{n}-Z_{h}^{n}\right\|_{H^{k}(\Omega)}
\end{aligned}
$$

where $\widetilde{Z}(s)$ satisfies the following ordinary differential equation

$$
\frac{\mathrm{d} \widetilde{Z}}{\mathrm{~d} s}(s)=F_{h}(s, \widetilde{Z}(s)),
$$

with the final condition $\widetilde{Z}\left(t^{n+1}\right)=z$, and where

$$
F_{h}(t, \cdot)=\sum_{n=0}^{N_{T}-1} F_{h}^{n}(\cdot) \chi_{n}(t)
$$

with $\chi_{n}(t)=1$ if $t \in\left[t^{n}, t^{n+1}[\right.$ and zero elsewhere.

Let us start with the estimate of $\left\|\widetilde{Z}^{n}-Z_{h}^{n}\right\|_{H^{k}(\Omega)}$. Let us first show that $\max _{n \leq N_{T}}\left\|\partial_{z} \widetilde{Z}^{n}\right\|_{L^{\infty}(\Omega)}$ is bounded. If we differentiate with respect to $z$ the equation

$$
\widetilde{Z}(t)=z+\int_{t^{n+1}}^{t} F_{h}(t, \widetilde{Z}(s)) \mathrm{d} s
$$

using a Gronwall lemma, we then get

$$
\left\|\partial_{z} \widetilde{Z}\right\|_{L^{\infty}\left(t^{n}, t^{n+1} ; L^{\infty}(\Omega)\right)} \leq \mathrm{e}^{\Delta t\left\|\nabla F_{h}\right\|_{L^{\infty}\left(0, T ; L^{\infty}(\Omega)\right)}},
$$


which is bounded because $\left\|\nabla F_{h}\right\|_{L^{\infty}\left(0, T ; L^{\infty}(\Omega)\right)}$ is also bounded. Using a second-order Taylor expansion of $\widetilde{Z}^{n}$ around $t=t^{n+1}$, and subtracting it to (3.6) there exists a time $\left.t^{\star} \in\right] t^{n}, t^{n+1}[$ such that

$$
0=Z_{h}^{n}-\widetilde{Z}^{n}+\Delta t\left(F_{h}^{n}\left(Z_{h}^{n}\right)-F_{h}^{n}\left(\widetilde{Z}^{n}\right)\right)+\frac{\Delta t^{2}}{2} \frac{\mathrm{d}^{2} \widetilde{Z}}{\mathrm{~d} t^{2}}\left(t^{\star}\right)
$$

Since we have

$$
\begin{aligned}
\frac{\mathrm{d}^{2} \widetilde{Z}}{\mathrm{~d} t^{2}}\left(t^{\star}\right) & =\partial_{t} F_{h}\left(t^{\star}, \widetilde{Z}\left(t^{\star}\right)\right)+\partial_{z} F_{h}^{n}\left(\widetilde{Z}\left(t^{\star}\right)\right) \partial_{z} \widetilde{Z}\left(t^{\star}\right) \\
& =\partial_{z} F_{h}^{n}\left(\widetilde{Z}\left(t^{\star}\right)\right) \partial_{z} \widetilde{Z}\left(t^{\star}\right),
\end{aligned}
$$

using (5.15), we get for $k=0,1$,

$$
\begin{aligned}
\left\|Z_{h}^{n}-\widetilde{Z}^{n}\right\|_{H^{k}(\Omega)} & \leq \Delta t\left\|F_{h}^{n}\left(Z_{h}^{n}\right)-F_{h}^{n}\left(\widetilde{Z}^{n}\right)\right\|_{H^{k}(\Omega)}+\frac{\Delta t^{2}}{2}\left\|\partial_{z} F_{h}^{n}\left(\widetilde{Z}\left(t^{\star}\right)\right)\right\|_{H^{k}(\Omega)}\left\|\partial_{z} \widetilde{Z}\left(t^{\star}\right)\right\|_{L^{\infty}(\Omega)} \\
& \lesssim \Delta t\left\|F_{h}^{n}\left(Z_{h}^{n}\right)-F_{h}^{n}\left(\widetilde{Z}^{n}\right)\right\|_{H^{k}(\Omega)}+C \Delta t^{2} \\
& \leq \Delta t \widetilde{C}\left(\sup _{n \leq N_{T}}\left\|f_{h}^{n}\right\|_{H^{2}(\Omega)}\right)\left(\left\|Z_{h}^{n}-\widetilde{Z}^{n}\right\|_{H^{k}(\Omega)}+\left\|Z_{h}^{n}-\widetilde{Z}^{n}\right\|_{H^{k}(\Omega)}^{1 / 2}\right)+C \Delta t^{2} .
\end{aligned}
$$

If $\Delta t$ is small enough, the above leads to

$$
\left((1-\widetilde{C} \Delta t)^{1 / 2}\left\|Z_{h}^{n}-\widetilde{Z}^{n}\right\|_{H^{k}(\Omega)}^{1 / 2}-\frac{\widetilde{C} \Delta t}{2(1-\widetilde{C} \Delta t)^{1 / 2}}\right)^{2} \leq\left(C+\frac{\widetilde{C}^{2}}{4(1-\widetilde{C} \Delta t)}\right) \Delta t^{2}
$$

which implies

$$
\left\|Z_{h}^{n}-\widetilde{Z}^{n}\right\|_{H^{k}(\Omega)} \leq\left(\sqrt{C+\frac{\widetilde{C}^{2}}{4(1-\widetilde{C} \Delta t)}}+\frac{\widetilde{C}}{2(1-\widetilde{C} \Delta t)^{1 / 2}}\right)^{2} \frac{\Delta t^{2}}{1-\widetilde{C} \Delta t} \lesssim \Delta t^{2},
$$

and ends the estimate of the term $\left\|\widetilde{Z}^{n}-Z_{h}^{n}\right\|_{H^{k}(\Omega)}$.

Let us now estimate the term $\left\|\widetilde{Z}^{n}-Z^{n}\right\|_{H^{k}(\Omega)}$. Using (2.17) and (5.14) we have

$$
Z(t)-\widetilde{Z}(t)=\int_{t^{n+1}}^{t}\left[(F(s, Z(s))-F(s, \widetilde{Z}(s)))+\left(F(s, \widetilde{Z}(s))-F_{h}(s, \widetilde{Z}(s))\right)\right] \mathrm{d} s .
$$

By taking the $H^{k}$-norm in the $z$ variable we get

$$
\begin{aligned}
\|Z(t)-\widetilde{Z}(t)\|_{H^{k}(\Omega)} \leq & \|F\|_{L^{\infty}\left(0, T ; W^{2, \infty}(\Omega)\right)} \int_{t^{n+1}}^{t}\|Z(s)-\widetilde{Z}(s)\|_{H^{k}(\Omega)} \\
& +\left\|\operatorname{det}^{-1} \partial_{z} \widetilde{Z}\right\|_{L^{\infty}([0, T] \times \Omega)}\left\|\partial_{z} \widetilde{Z}\right\|_{L^{\infty}([0, T] \times \Omega)} \int_{t^{n+1}}^{t}\left\|F(s)-F_{h}(s)\right\|_{H^{k}(\Omega)} \mathrm{d} s .
\end{aligned}
$$

By taking the $L^{\infty}$-norm in the $t$ variable on the time interval $\left[t^{n}, t^{n+1}\right]$ we get

$$
\|Z-\widetilde{Z}\|_{L^{\infty}\left(t^{n}, t^{n+1} ; H^{k}(\Omega)\right)} \lesssim \Delta t\|Z-\widetilde{Z}\|_{L^{\infty}\left(t^{n}, t^{n+1} ; H^{k}(\Omega)\right)}+\Delta t\left\|F-F_{h}\right\|_{L^{\infty}\left(t^{n}, t^{n+1} ; H^{k}(\Omega)\right)} .
$$


If $\Delta t$ is small enough then

$$
\begin{aligned}
\left\|Z^{n}-\widetilde{Z}^{n}\right\|_{H^{k}(\Omega)} & \leq\|Z-\widetilde{Z}\|_{L^{\infty}\left(t^{n}, t^{n+1} ; H^{k}(\Omega)\right)} \\
& \leq \frac{C \Delta t}{1-\Delta t}\left\|F-F_{h}\right\|_{L^{\infty}\left(t^{n}, t^{n+1} ; H^{k}(\Omega)\right)}
\end{aligned}
$$

Using a Taylor expansion around the time $t$, there exists $\left.t^{\star} \in\right] t^{n}, t^{n+1}[$ such that

$$
\left(F-F_{h}\right)(t)=\left(F-F_{h}\right)\left(t^{n}\right)-\left(t-t^{n}\right) \partial_{t} F\left(t^{\star}\right) .
$$

By taking the norm of the previous equation, we get

$$
\left\|F-F_{h}\right\|_{L^{\infty}\left(t^{n}, t^{n+1} ; H^{k}(\Omega)\right)} \leq\left\|F^{n}-F_{h}^{n}\right\|_{H^{k}(\Omega)}+\Delta t\left\|\partial_{t} F\right\|_{L^{\infty}\left(0, T ; H^{k}(\Omega)\right)} .
$$

From the above and the estimate (5.17), we obtain

$$
\left\|Z^{n}-\widetilde{Z}^{n}\right\|_{H^{k}(\Omega)} \lesssim \Delta t^{2}+\Delta t\left\|F^{n}-F_{h}^{n}\right\|_{H^{k}(\Omega)}
$$

From the definition of $F$ and $F_{h}^{n}$, using Proposition 5.1, the bounds (5.1) and Lemma 2.3, we get

$$
\left\|F^{n}-F_{h}^{n}\right\|_{H^{k}(\Omega)} \lesssim\left\|\mu_{h}^{n}-\mu^{n}\right\|_{H^{k}\left(\Omega_{r}\right)}+\left\|\mu_{h}^{n \prime}-\mu^{n \prime}\right\|_{H^{k}\left(\Omega_{r}\right)}+\left\|\lambda_{h}^{n}-\lambda^{n}\right\|_{H^{k}\left(\Omega_{r}\right)}+\left\|\dot{\lambda}_{h}^{n}-\dot{\lambda}^{n}\right\|_{H^{k}\left(\Omega_{r}\right)} .
$$

Using the integrated Einstein equations (2.5)-(2.7) and (2.12), the bounds of the characteristics (5.3) and Lemma 2.3, the inequality (5.19) becomes

$$
\left\|F^{n}-F_{h}^{n}\right\|_{H^{k}(\Omega)} \lesssim\left\|j_{h}^{n}-j^{n}\right\|_{H^{k}\left(\Omega_{r}\right)}+\left\|\rho_{h}^{n}-\rho^{n}\right\|_{H^{k}\left(\Omega_{r}\right)}+\left\|p_{h}^{n}-p^{n}\right\|_{H^{k}\left(\Omega_{r}\right)}+\left\|m_{h}^{n}-m^{n}\right\|_{H^{k}\left(\Omega_{r}\right)} .
$$

Using the estimate (5.3), Lemma 2.3 and the definition of the moments (2.8)-(2.10) and (2.13), equation (5.20) becomes

$$
\left\|F^{n}-F_{h}^{n}\right\|_{H^{k}(\Omega)} \lesssim\left\|f^{n}-f_{h}^{n}\right\|_{H^{k}(\Omega)} .
$$

Equations (5.21) and (5.18) lead to

$$
\left\|Z^{n}-\widetilde{Z}^{n}\right\|_{H^{k}(\Omega)} \lesssim \Delta t^{2}+\Delta t\left\|f^{n}-f_{h}^{n}\right\|_{H^{k}(\Omega)},
$$

which concludes the estimate. By putting together the estimates (5.22), (5.13) and (5.16), we get the desired result.

Now we are ready to prove Theorem 4.1.

Proof of Theorem 4.1. Assembling Lemmas 5.3, 5.4 and the estimate (5.2), we obtain for the global error

$$
E^{n+1} \leq C\left(h^{m-k}+\Delta t^{2}\right)+(1+C \Delta t) E^{n}
$$

By a Gronwall lemma, we obtain for $n \leq N_{T_{h}}$ and $k=0,1$,

$$
\left\|f^{n}-f_{h}^{n}\right\|_{H^{k}(\Omega)} \lesssim \Delta t+\frac{h^{m-k}}{\Delta t} .
$$


From the estimate (5.23) and the following inequalities

$$
\begin{aligned}
& \left\|\mu_{h}^{n}-\mu^{n}\right\|_{L^{2}\left(\Omega_{r}\right)},\left\|\mu_{h}^{n \prime}-\mu^{n \prime}\right\|_{L^{2}\left(\Omega_{r}\right)},\left\|\lambda_{h}^{n}-\lambda^{n}\right\|_{L^{2}\left(\Omega_{r}\right)}, \\
& \left\|\lambda_{h}^{n \prime}-\lambda^{n \prime}\right\|_{L^{2}\left(\Omega_{r}\right)},\left\|m_{h}^{n}-m^{n}\right\|_{L^{2}\left(\Omega_{r}\right)},\left\|m_{h}^{n \prime}-m^{n \prime}\right\|_{L^{2}\left(\Omega_{r}\right)} \lesssim\left\|f^{n}-f_{h}^{n}\right\|_{L^{2}(\Omega)}
\end{aligned}
$$

we get the second error estimate of Theorem 4.1. Using the estimate (5.23) and the following inequalities

$$
\left\|\rho_{h}^{n}-\rho^{n}\right\|_{L^{2}\left(\Omega_{r}\right)},\left\|p_{h}^{n}-p^{n}\right\|_{L^{2}\left(\Omega_{r}\right)},\left\|j_{h}^{n}-j^{n}\right\|_{L^{2}\left(\Omega_{r}\right)} \lesssim\left\|f^{n}-f_{h}^{n}\right\|_{L^{2}(\Omega)}
$$

we obtain the third error estimates of Theorem 4.1.

Now it remains to establish the assertions on the length $T_{h}$ of our approximation interval. Let us choose $h$ and $\Delta t$ such that

$$
\Delta t+\frac{h^{m-1}}{\Delta t}+\frac{h^{m}}{\Delta t} \leq \frac{K}{2} \quad \text { and } \quad \Delta t+\frac{h^{m}}{\Delta t} \leq \frac{r_{\min }}{2} \frac{(I-J)}{I J}
$$

with $K$ being the constant of Lemma 2.3 and $I, J \in \mathbb{R}$ such that $1<J<I$. We then have

$$
\begin{gathered}
\left\|\mu_{h}\right\|_{L^{\infty}\left([0, T] \times \Omega_{r}\right)} \leq\|\mu\|_{L^{\infty}\left([0, T] \times \Omega_{r}\right)}+\sup _{n \leq N_{T}}\left\|\mu_{h}^{n}-\mu^{n}\right\|_{L^{\infty}\left(\Omega_{r}\right)} \leq K+\frac{K}{2}=\frac{3 K}{2}, \\
\left\|\lambda_{h}\right\|_{L^{\infty}\left([0, T] \times \Omega_{r}\right)} \leq\|\lambda\|_{L^{\infty}\left([0, T] \times \Omega_{r}\right)}+\sup _{n \leq N_{T}}\left\|\lambda_{h}^{n}-\lambda^{n}\right\|_{L^{\infty}\left(\Omega_{r}\right)} \leq K+\frac{K}{2}=\frac{3 K}{2}, \\
\left\|\mu_{h}^{\prime}\right\|_{L^{\infty}\left([0, T] \times \Omega_{r}\right)} \leq\left\|\mu^{\prime}\right\|_{L^{\infty}\left([0, T] \times \Omega_{r}\right)}+\sup _{n \leq N_{T}}\left\|\mu_{h}^{n \prime}-\mu^{n \prime}\right\|_{L^{\infty}\left(\Omega_{r}\right)} \leq K+\frac{K}{2}=\frac{3 K}{2} \\
\left\|\lambda_{h}^{\prime}\right\|_{L^{\infty}\left([0, T] \times \Omega_{r}\right)} \leq\left\|\lambda^{\prime}\right\|_{L^{\infty}\left([0, T] \times \Omega_{r}\right)}+\sup _{n \leq N_{T}}\left\|\lambda_{h}^{n \prime}-\lambda^{n \prime}\right\|_{L^{\infty}\left(\Omega_{r}\right)} \leq K+\frac{K}{2}=\frac{3 K}{2}, \\
\left\|\dot{\lambda}_{h}\right\|_{L^{\infty}\left([0, T] \times \Omega_{r}\right)} \leq\|\dot{\lambda}\|_{L^{\infty}\left([0, T] \times \Omega_{r}\right)}+\sup _{n \leq N_{T}}\left\|\dot{\lambda}_{h}^{n}-\dot{\lambda}^{n}\right\|_{L^{\infty}\left(\Omega_{r}\right)} \leq K+\frac{K}{2}=\frac{3 K}{2} .
\end{gathered}
$$

Using the property (2.20) we obtain

$$
\begin{aligned}
m_{h}^{n}(r) & =m\left(t^{n}, r\right)+\left(m_{h}^{n}(r)-m\left(t^{n}, r\right)\right) \\
& \leq \frac{r}{2 I}+\Delta t+\frac{h^{m}}{\Delta t}<\frac{r}{2 I}+\frac{r}{2} \frac{(I-J)}{I J}<\frac{r}{2 J} .
\end{aligned}
$$

Thus $T_{h}=T$ since otherwise the approximation interval for (5.1) could be extended beyond $T_{h}$.

\subsection{Convergence of the second-order in time scheme}

The error terms $E 1$ and $E 3$ are of the same type as in the first-order scheme and the proofs can be handled in the same way. In fact, the proof is identical for the term $E 1$ because it is just an interpolation error which depends only on the approximation property (3.2) of the reconstruction operator $\Pi_{h}$ and does not depend of the time discretization scheme. For the accumulation error E3, we apply successively to each stage of the second-order two-stage Runge-Kutta scheme (which can be viewed as a succession of two first-order one-stage Runge-Kutta scheme) stability analysis which have been done for the first-order one-stage Runge-Kutta scheme. In fact for the second (resp. the first) stage of the second-order Runge-Kutta scheme defined in Section 3.1.2, by using equation (3.10) (resp. (3.8)) instead of equation (3.6) and following the proof of Proposition 5.1 which uses equation (3.6), we can show that the estimates (5.3) (resp. estimates (5.3) where we replace $Z_{h}^{n}$ by $\widehat{Z}_{h}^{n}$ ) still hold. In order to get $H^{3}$-stability of the scheme, we proceed as for the proof of Lemma 5.1. The idea is to control 
the norm $\left\|f_{h}^{n+1}\right\|_{H^{3}(\Omega)}$ by the norm $\left\|f_{h}^{n}\right\|_{H^{3}(\Omega)}$, thanks to the estimate of the partial derivatives of characteristic curves $\partial_{z}^{\alpha} Z_{h}^{n}$ (with $|\alpha| \leq 3$ ). Following the proof of Lemma 5.1, from equation (3.10), we can control the partial derivatives $\partial_{z}^{\alpha} Z_{h}^{n}$ (with $|\alpha| \leq 3$ ) by the derivatives of the approximate force field $F_{h}^{n+1 / 2}$ which can be controlled by the term $\left\|f_{h}^{n+1 / 2}\right\|_{H^{3}(\Omega)}$ thanks to a lemma of the same type of Lemma 5.2. Using equation (3.8), we can control $\left\|f_{h}^{n+1 / 2}\right\|_{H^{3}(\Omega)}$ by the partial derivatives $\partial_{z}^{\alpha} \widehat{Z}_{h}^{n}$ (with $|\alpha| \leq 3$ ) which can be controlled by the derivatives of the approximate force field $F_{h}^{n}$ and consequently by the norm $\left\|f_{h}^{n}\right\|_{H^{3}(\Omega)}$. Therefore we can control the norm $\left\|f_{h}^{n+1}\right\|_{H^{3}(\Omega)}$ by the norm $\left\|f_{h}^{n}\right\|_{H^{3}(\Omega)}$ and we obtain the $H^{3}$-stability of the scheme. Following the proof of Lemma 5.3, the $H^{3}$-stability property allows to get the same estimate as the one obtained for the first-order scheme for the error term E3. Therefore the convergence proof for the second-order scheme differs from the treatment of the error term E2. In fact, second-order error estimates in time arise from this term E2. The estimate (5.13) and the equation (5.14) are still valid but we now have

$$
\begin{aligned}
F_{h}(t, \cdot)= & \sum_{n=0}^{N_{T}-1}\left(\frac{t^{n+1 / 2}-t}{\Delta t / 2} F_{h}^{n}(\cdot)+\frac{t-t^{n}}{\Delta t / 2} F_{h}^{n+1 / 2}(\cdot)\right) \chi_{n}(t) \\
& +\left(\frac{t-t^{n+1 / 2}}{\Delta t / 2} F_{h}^{n+1}(\cdot)+\frac{t^{n+1}-t}{\Delta t / 2} F_{h}^{n+1 / 2}(\cdot)\right) \chi_{n+1 / 2}(t)
\end{aligned}
$$

where $\chi_{n}(t)=1$ if $t \in\left[t^{n}, t^{n+1 / 2}\right.$ [ and zero elsewhere, and $\chi_{n+1 / 2}(t)=1$ if $t \in\left[t^{n+1 / 2}, t^{n+1}\right.$ [ and zero elsewhere.

Let us deal first with the estimate of the term $\left\|Z^{n}-\widetilde{Z}^{n}\right\|_{H^{k}(\Omega)}$. Let the time $t \in\left[t^{n}, t^{n+1 / 2}[\right.$. Using a Taylor expansion around the time $t$, there exist $\left.t^{\dagger}, t^{\ddagger} \in\right] t^{n}, t^{n+1 / 2}[$ such that

$$
\left(F_{h}-F\right)\left(t^{n}\right)=\left(F_{h}-F\right)(t)+\left(t^{n}-t\right) \partial_{t}\left(F-F_{h}\right)(t)+\frac{\left(t^{n}-t\right)^{2}}{2} \partial_{t}^{2}\left(F_{h}-F\right)\left(t^{\dagger}\right)
$$

and

$$
\left(F_{h}-F\right)\left(t^{n+1 / 2}\right)=\left(F_{h}-F\right)(t)+\left(t^{n+1 / 2}-t\right) \partial_{t}\left(F-F_{h}\right)(t)+\frac{\left(t^{n+1 / 2}-t\right)^{2}}{2} \partial_{t}^{2}\left(F_{h}-F\right)\left(t^{\ddagger}\right) .
$$

If we multiply equation $(5.25)$ by $2\left(t^{n+1 / 2}-t\right) / \Delta t$, equation $(5.26)$ by $2\left(t-t^{n}\right) / \Delta t$, and add the two expressions, after taking the norm $\|\cdot\|_{L^{\infty}\left(\left[t^{n}, t^{n+1 / 2}\left[; H^{k}(\Omega)\right)\right.\right.}$ we get

$$
\left\|F_{h}-F\right\|_{L^{\infty}\left(\left[t^{n}, t^{n+1 / 2}\left[; H^{k}(\Omega)\right)\right.\right.} \leq\left\|F_{h}^{n}-F^{n}\right\|_{H^{k}(\Omega)}+\left\|F_{h}^{n+1 / 2}-F^{n+1 / 2}\right\|_{H^{k}(\Omega)}+\Delta t^{2}\left\|\partial_{t}^{2} F\right\|_{L^{\infty}\left(0, T ; H^{k}(\Omega)\right)}
$$

where we have used the fact that $\left.\partial_{t}^{2} F_{h}=0, \forall t \in\right] t^{n}, t^{n+1 / 2}[$. In the same way we get

$$
\left\|F_{h}-F\right\|_{L^{\infty}\left(\left[t^{n+1 / 2}, t^{n+1}\left[; H^{k}(\Omega)\right)\right.\right.} \leq\left\|F_{h}^{n+1}-F^{n+1}\right\|_{H^{k}(\Omega)}+\left\|F_{h}^{n+1 / 2}-F^{n+1 / 2}\right\|_{H^{k}(\Omega)}+\Delta t^{2}\left\|\partial_{t}^{2} F\right\|_{L^{\infty}\left(0, T ; H^{k}(\Omega)\right)} .
$$

Finally we obtain

$$
\begin{aligned}
\left\|F_{h}-F\right\|_{L^{\infty}\left(\left[t^{n}, t^{n+1}\left[; H^{k}(\Omega)\right) \leq\right.\right.} & \left\|F_{h}^{n}-F^{n}\right\|_{H^{k}(\Omega)}+\left\|F_{h}^{n+1}-F^{n+1}\right\|_{H^{k}(\Omega)} \\
& +2\left\|F_{h}^{n+1 / 2}-F^{n+1 / 2}\right\|_{H^{k}(\Omega)}+\mathcal{O}\left(\Delta t^{2}\right) .
\end{aligned}
$$


From what we have done for the first-order scheme we get

$$
\begin{aligned}
\left\|F_{h}^{n+1}-F^{n+1}\right\|_{H^{k}(\Omega)} & \lesssim\left\|f_{h}^{n+1}-f^{n+1}\right\|_{H^{k}(\Omega)}, \\
\left\|F_{h}^{n}-F^{n}\right\|_{H^{k}(\Omega)} & \lesssim\left\|f_{h}^{n}-f^{n}\right\|_{H^{k}(\Omega)}, \\
\left\|F_{h}^{n+1 / 2}-F^{n+1 / 2}\right\|_{H^{k}(\Omega)} & \lesssim\left\|f_{h}^{n+1 / 2}-f^{n+1 / 2}\right\|_{H^{k}(\Omega)} \\
& \lesssim h^{m-k}+\Delta t^{2}+(1+C \Delta t)\left\|f_{h}^{n}-f^{n}\right\|_{H^{k}(\Omega)},
\end{aligned}
$$

so that in the end we get the estimate

$$
\left\|Z^{n}-\widetilde{Z}^{n}\right\|_{H^{k}(\Omega)} \lesssim \Delta t^{3}+h^{m-k} \Delta t+\Delta t\left(\left\|f_{h}^{n}-f^{n}\right\|_{H^{k}(\Omega)}+\left\|f_{h}^{n+1}-f^{n+1}\right\|_{H^{k}(\Omega)}\right) .
$$

Let us deal now with the term $\left\|\widetilde{Z}^{n}-Z_{h}^{n}\right\|_{H^{k}(\Omega)}$. Using a Taylor expansion around the time $t^{n+1 / 2}$, there exist times $\left.t^{\dagger}, t^{\ddagger} \in\right] t^{n}, t^{n+1}[$ such that

$$
\widetilde{Z}^{n}=\widetilde{Z}^{n+1 / 2}-\frac{\Delta t}{2} \frac{\mathrm{d}}{\mathrm{d} t} \widetilde{Z}^{n+1 / 2}+\frac{\Delta t^{2}}{8} \frac{\mathrm{d}^{2}}{\mathrm{~d} t^{2}} \widetilde{Z}^{n+1 / 2}-\frac{\Delta t^{3}}{48} \frac{\mathrm{d}^{3}}{\mathrm{~d} t^{3}} \widetilde{Z}^{\dagger}
$$

and

$$
\widetilde{Z}^{n+1}=\widetilde{Z}^{n+1 / 2}+\frac{\Delta t}{2} \frac{\mathrm{d}}{\mathrm{d} t} \widetilde{Z}^{n+1 / 2}+\frac{\Delta t^{2}}{8} \frac{\mathrm{d}^{2}}{\mathrm{~d} t^{2}} \widetilde{Z}^{n+1 / 2}+\frac{\Delta t^{3}}{48} \frac{\mathrm{d}^{3}}{\mathrm{~d} t^{3}} \widetilde{Z}^{\ddagger} .
$$

If we subtract the expressions (5.30) and (5.29) we get

$$
\widetilde{Z}^{n+1}-\widetilde{Z}^{n}=\Delta t \frac{\mathrm{d}}{\mathrm{d} t} \widetilde{Z}^{n+1 / 2}+\frac{\Delta t^{3}}{48}\left(\frac{\mathrm{d}^{3}}{\mathrm{~d} t^{3}} \widetilde{Z}^{\dagger}+\frac{\mathrm{d}^{3}}{\mathrm{~d} t^{3}} \widetilde{Z}^{\ddagger}\right) .
$$

Using the second order scheme (3.10)

$$
Z_{h}^{n+1}-Z_{h}^{n}=\Delta t F_{h}^{n+1 / 2}\left(\frac{Z_{h}^{n}+z}{2}\right)
$$

and the equation

$$
\frac{\mathrm{d}}{\mathrm{d} t} \widetilde{Z}^{n+1 / 2}=F_{h}\left(t^{n+1 / 2}, \widetilde{Z}^{n+1 / 2}\right)=F_{h}^{n+1 / 2}\left(\widetilde{Z}^{n+1 / 2}\right),
$$

and by noting that $Z_{h}^{n+1}=\widetilde{Z}^{n+1}=z$, the difference of (5.31) and (5.32) gives

$$
0=\widetilde{Z}^{n}-Z_{h}^{n}+\Delta t\left(F_{h}^{n+1 / 2}\left(\widetilde{Z}^{n+1 / 2}\right)-F_{h}^{n+1 / 2}\left(\frac{Z_{h}^{n}+z}{2}\right)\right)+\frac{\Delta t^{3}}{48}\left(\frac{\mathrm{d}^{3}}{\mathrm{~d} t^{3}} \widetilde{Z}^{\dagger}+\frac{\mathrm{d}^{3}}{\mathrm{~d} t^{3}} \widetilde{Z}^{\ddagger}\right) .
$$

The sum $((5.29)+(5.30)) / 2$ gives

$$
\frac{\widetilde{Z}^{n+1}+\widetilde{Z}^{n}}{2}=\widetilde{Z}^{n+1 / 2}+\frac{\Delta t^{2}}{16}\left(\frac{\mathrm{d}^{2}}{\mathrm{~d} t^{2}} \widetilde{Z}^{\natural}+\frac{\mathrm{d}^{2}}{\mathrm{~d} t^{2}} \widetilde{Z}^{\sharp}\right)
$$


Using equations (5.33) and (5.34) and taking the norm $\|\cdot\|_{H^{k}(\Omega)}$ we get

$$
\begin{aligned}
\left\|\widetilde{Z}^{n}-Z_{h}^{n}\right\|_{H^{k}(\Omega)} \lesssim & \Delta t\left\|F_{h}^{n+1 / 2}\right\|_{H^{3}(\Omega)}\left\|\widetilde{Z}^{n}-Z_{h}^{n}\right\|_{H^{k}(\Omega)}+\Delta t^{3}\left\|F_{h}^{n+1 / 2}\right\|_{H^{3}(\Omega)}\left\|\frac{\mathrm{d}^{2}}{\mathrm{~d} t^{2}} \widetilde{Z}^{\natural}+\frac{\mathrm{d}^{2}}{\mathrm{~d} t^{2}} \widetilde{Z}^{\sharp}\right\|_{H^{k}(\Omega)} \\
& +\Delta t^{3}\left\|F_{h}^{n+1 / 2}\right\|_{H^{3}(\Omega)}\left\|\frac{\mathrm{d}^{3}}{\mathrm{~d} t^{3}} \widetilde{Z}^{\dagger}+\frac{\mathrm{d}^{3}}{\mathrm{~d} t^{3}} \widetilde{Z}^{\ddagger}\right\|_{H^{k}(\Omega)} \\
\leq & \Delta t C\left(\sup _{n \leq N_{T}}\left\|f_{h}^{n}\right\|_{H^{3}(\Omega)}\right)\left(\left\|\widetilde{Z}^{n}-Z_{h}^{n}\right\|_{H^{k}(\Omega)}+\Delta t^{2}\left\{\left\|\frac{\mathrm{d}^{2}}{\mathrm{~d} t^{2}} \widetilde{Z}^{\natural}+\frac{\mathrm{d}^{2}}{\mathrm{~d} t^{2}} \widetilde{Z}^{\sharp}\right\|_{H^{k}(\Omega)}\right.\right. \\
& \left.\left.+\left\|\frac{\mathrm{d}^{3}}{\mathrm{~d} t^{3}} \widetilde{Z}^{\dagger}+\frac{\mathrm{d}^{3}}{\mathrm{~d} t^{3}} \widetilde{Z}^{\ddagger}\right\|_{H^{k}(\Omega)}\right\}\right) .
\end{aligned}
$$

From estimate of the last two terms of the right hand side of the inequality (5.35) given in Appendix B and assuming that $\Delta t / h$ is bounded, an estimate of the term $\left\|\widetilde{Z}^{n}-Z_{h}^{n}\right\|_{H^{k}(\Omega)}$ gives

$$
\begin{aligned}
\left\|\widetilde{Z}^{n}-Z_{h}^{n}\right\|_{H^{k}(\Omega)} \lesssim & \Delta t\left\|\widetilde{Z}^{n}-Z_{h}^{n}\right\|_{H^{k}(\Omega)}+\Delta t^{3} \\
& +\Delta t\left(\left\|f_{h}^{n}-f^{n}\right\|_{H^{k}(\Omega)}+\left\|f_{h}^{n+1 / 2}-f^{n+1 / 2}\right\|_{H^{k}(\Omega)}+\left\|f_{h}^{n+1}-f^{n+1}\right\|_{H^{k}(\Omega)}\right) \\
& \lesssim \Delta t^{3}+h^{m-k} \Delta t+\Delta t\left(\left\|f_{h}^{n}-f^{n}\right\|_{H^{k}(\Omega)}+\left\|f_{h}^{n+1}-f^{n+1}\right\|_{H^{k}(\Omega)}\right) .
\end{aligned}
$$

Finally the global error $E^{n+1}$ has the following bound

$$
\begin{aligned}
E^{n+1} & \leq C\left(h^{m-k}+\Delta t^{3}+h^{m-k} \Delta t\right)+\left(1+C \Delta t E^{n}\right)+C \Delta t E^{n+1} \\
& \leq \frac{C}{1-C \Delta t}\left(h^{m-k}+\Delta t^{3}+h^{m-k} \Delta t\right)+\left(\frac{1+C \Delta t}{1-C \Delta t}\right) E^{n} \\
& \lesssim \frac{h^{m-k}}{\Delta t}+\Delta t^{2}+h^{m-k}+\left(\frac{1+C \Delta t}{1-C \Delta t}\right)^{N_{T}} E^{0} \\
& \lesssim \Delta t^{2}+\frac{h^{m-k}}{\Delta t}
\end{aligned}
$$

if we suppose that $E^{0}=\mathcal{O}\left(h^{m-k}\right)$. The end of the proof is the same as for the first order.

\section{Conclusion}

In this paper we have presented the convergence analysis and a priori error estimates of a high-order semiLagrangian scheme to solve the reduced Vlasov-Einstein system for the spherically symmetric asymptotically flat case in Schwarzschild coordinates. This work is the starting point for further studies. In fact, following what has been done in [10], we shall modify the present scheme to obtain an adaptive semi-Lagrangian scheme allowing to solve the reduced Vlasov-Einstein for the spherically symmetric asymptotically flat case system in Schwarzschild and/or maximal-areal coordinates. Therefore we can numerically study critical collapse phenomena for collisionless matter and compare the results with other similar simulations $[2,14,24]$. 


\section{A. Proof of Lemma 5.2}

Proof. It follows from the definition of $\left(F_{h, r}^{n}, F_{h, w}^{n}\right)$, obtained by the discretization of $(2.14)$ and $(2.15)$, the bounds

$$
\begin{aligned}
\left\|\partial_{r}^{m} \partial_{w}^{\alpha} \partial_{l}^{\beta} F_{h, r}^{n}\right\|_{L^{\infty}(\Omega)} & \leq C \begin{cases}1 & \text { if } m \leq 1 \\
\left\|\mu_{h}^{n^{(m)}}\right\|_{L^{\infty}\left(\Omega_{r}\right)}+\left\|\lambda_{h}^{n^{(m)}}\right\|_{L^{\infty}\left(\Omega_{r}\right)} & \text { if } m>1\end{cases} \\
\leq & C\left(\left\|p_{h}^{n^{(m-1)}}\right\|_{L^{\infty}\left(\Omega_{r}\right)}+\left\|\rho_{h}^{n^{(m-1)}}\right\|_{L^{\infty}\left(\Omega_{r}\right)}\right) \\
\leq & C\left(\left\|\rho_{h}^{n}\right\|_{H^{m}\left(\Omega_{r}\right)}+\left\|p_{h}^{n}\right\|_{H^{m}\left(\Omega_{r}\right)}\right) \\
\leq & C\left\|f_{h}^{n}\right\|_{H^{m}(\Omega)} . \\
\left\|\partial_{r}^{m} \partial_{w}^{\alpha} \partial_{l}^{\beta} F_{h, w}^{n}\right\|_{L^{\infty}(\Omega)} \leq C\left(\left\|\mu_{h}^{n^{(m)}}\right\|_{L^{\infty}\left(\Omega_{r}\right)}+\left\|\lambda_{h}^{n^{(m)}}\right\|_{L^{\infty}\left(\Omega_{r}\right)}+\left\|\mu_{h}^{n^{(m+1)}}\right\|_{L^{\infty}\left(\Omega_{r}\right)}+\left\|\dot{\lambda}_{h}^{n^{(m)}}\right\|_{L^{\infty}\left(\Omega_{r}\right)}\right) & \\
& \leq C\left(\left\|p_{h}^{n^{(m-1)}}\right\|_{L^{\infty}\left(\Omega_{r}\right)}+\left\|\rho_{h}^{n^{(m-1)}}\right\|_{L^{\infty}\left(\Omega_{r}\right)}+\left\|p_{h}^{n^{(m)}}\right\|_{L^{\infty}\left(\Omega_{r}\right)}+\left\|j_{h}^{n^{(m)}}\right\|_{L^{\infty}\left(\Omega_{r}\right)}\right) \\
& \leq C\left(\left\|\rho_{h}^{n}\right\|_{H^{m}\left(\Omega_{r}\right)}+\left\|p_{h}^{n}\right\|_{H^{m+1}\left(\Omega_{r}\right)}+\left\|j_{h}^{n}\right\|_{H^{m+1}\left(\Omega_{r}\right)}\right) \\
& \leq C\left\|f_{h}^{n}\right\|_{H^{m+1}(\Omega)} .
\end{aligned}
$$

Let us estimate the last two terms of the right hand side of the inequality (5.35). We first begin with the second term and we only give an estimate of the term $\left\|\frac{\mathrm{d}^{2}}{\mathrm{~d} t^{2}} \widetilde{Z}\right\|_{H^{k}(\Omega)}$. The proof is the same for the term $\left\|\frac{\mathrm{d}^{2}}{\mathrm{~d} t^{2}} \widetilde{Z}^{\sharp}\right\|_{H^{k}(\Omega)}$. We have

$$
\begin{aligned}
\left\|\frac{\mathrm{d}^{2}}{\mathrm{~d} t^{2}} \widetilde{Z}^{\natural}\right\|_{H^{k}(\Omega)} & \leq\left\|\partial_{t} F_{h}\left(t^{\natural}, \widetilde{Z}^{\natural}\right)\right\|_{H^{k}(\Omega)}+\left\|\nabla F_{h}\left(t^{\natural}, \widetilde{Z}^{\natural}\right) F_{h}\left(t^{\natural}, \widetilde{Z}^{\natural}\right)\right\|_{H^{k}(\Omega)} \\
& \lesssim\left\|\partial_{t} F_{h}\left(t^{\natural}, \widetilde{Z}^{\natural}\right)\right\|_{H^{k}(\Omega)}+\left\|F_{h}\left(t^{\natural}, \widetilde{Z}^{\natural}\right)\right\|_{H^{2}(\Omega)}^{2} \\
& \leq\left\|\partial_{t} F_{h}\left(t^{\natural}, \widetilde{Z}^{\natural}\right)\right\|_{H^{k}(\Omega)}+C\left(\sup _{n \leq N_{T}}\left\|f_{h}^{n}\right\|_{H^{2}(\Omega)}\right) .
\end{aligned}
$$

Let us estimate $\left\|\partial_{t} F_{h}\left(t^{\natural}, \widetilde{Z}^{\natural}\right)\right\|_{H^{k}(\Omega)}$. From the definition (5.24) we have

$$
\begin{aligned}
\left\|\partial_{t} F_{h}\left(t^{\natural}, \widetilde{Z}^{\natural}\right)\right\|_{H^{k}(\Omega)} \leq & \frac{2\left\|F_{h}^{n+1 / 2}\left(\widetilde{Z}^{\natural}\right)-F_{h}^{n}\left(\widetilde{Z}^{\natural}\right)\right\|_{H^{k}(\Omega)}}{\Delta t}+\frac{2\left\|F_{h}^{n+1}\left(\widetilde{Z}^{\natural}\right)-F_{h}^{n+1 / 2}\left(\widetilde{Z}^{\natural}\right)\right\|_{H^{k}(\Omega)}}{\Delta t} \\
\leq & \frac{2\left\|F_{h}^{n}\left(\widetilde{Z}^{\natural}\right)-F^{n}\left(\widetilde{Z}^{\natural}\right)\right\|_{H^{k}(\Omega)}}{\Delta t} \frac{2\left\|F_{h}^{n+1}\left(\widetilde{Z}^{\natural}\right)-F^{n+1}\left(\widetilde{Z}^{\natural}\right)\right\|_{H^{k}(\Omega)}}{\Delta t} \\
& +\frac{4\left\|F_{h}^{n+1 / 2}\left(\widetilde{Z}^{\natural}\right)-F^{n+1 / 2}\left(\widetilde{Z}^{\natural}\right)\right\|_{H^{k}(\Omega)}}{\Delta t}+\frac{2\left\|F^{n+1 / 2}\left(\widetilde{Z}^{\natural}\right)-F^{n}\left(\widetilde{Z}^{\natural}\right)\right\|_{H^{k}(\Omega)}}{\Delta t} \\
& +\frac{2\left\|F^{n+1 / 2}\left(\widetilde{Z}^{\natural}\right)-F^{n+1}\left(\widetilde{Z}^{\natural}\right)\right\|_{H^{k}(\Omega)}}{\Delta t} .
\end{aligned}
$$


Therefore we have

$$
\begin{aligned}
\left\|\partial_{t} F_{h}\left(t^{\natural}, \widetilde{Z}^{\natural}\right)\right\|_{H^{k}(\Omega)} \leq & \frac{C\left\|f_{h}^{n+1 / 2}-f^{n+1 / 2}\right\|_{H^{k}(\Omega)}}{\Delta t}+C\left\|\partial_{t} F\left(\cdot, \widetilde{Z}^{\natural}\right)\right\|_{L^{\infty}\left(0, T ; H^{k}(\Omega)\right)} \\
& +\frac{C\left\|f_{h}^{n}-f^{n}\right\|_{H^{k}(\Omega)}}{\Delta t}+\frac{C\left\|f_{h}^{n+1}-f^{n+1}\right\|_{H^{k}(\Omega)}}{\Delta t} .
\end{aligned}
$$

Now we only give an estimate of the term $\left\|\frac{\mathrm{d}^{3}}{\mathrm{~d} t^{3}} \widetilde{Z}^{\dagger}\right\|_{H^{k}(\Omega)}$, since the proof is the same for the term $\left\|\frac{\mathrm{d}^{3}}{\mathrm{~d} t^{3}} \widetilde{Z}^{\ddagger}\right\|_{H^{k}(\Omega)}$. We obtain

$$
\begin{aligned}
\left\|\frac{\mathrm{d}^{3}}{\mathrm{~d} t^{3}} \widetilde{Z}^{\dagger}\right\|_{H^{k}(\Omega)} \leq & \left\|\partial_{t}^{2} F_{h}\left(t^{\dagger}, \widetilde{Z}^{\dagger}\right)\right\|_{H^{k}(\Omega)}+2\left\|\nabla \partial_{t} F_{h}\left(t^{\dagger}, \widetilde{Z}^{\dagger}\right) F_{h}\left(t^{\dagger}, \widetilde{Z}^{\dagger}\right)\right\|_{H^{k}(\Omega)}+\left\|\nabla F_{h}\left(t^{\dagger}, \widetilde{Z}^{\dagger}\right) \partial_{t} F_{h}\left(t^{\dagger}, \widetilde{Z}^{\dagger}\right)\right\|_{H^{k}(\Omega)} \\
& +\left\|\nabla^{2} F_{h}\left(t^{\dagger}, \widetilde{Z}^{\dagger}\right)\left|F_{h}\left(t^{\dagger}, \widetilde{Z}^{\dagger}\right)\right|^{2}\right\|_{H^{k}(\Omega)}+\left\|\left|\nabla F_{h}\left(t^{\dagger}, \widetilde{Z}^{\dagger}\right)\right|^{2} F_{h}\left(t^{\dagger}, \widetilde{Z}^{\dagger}\right)\right\|_{H^{k}(\Omega)} \\
\lesssim & \left\|F_{h}\right\|_{L^{\infty}\left(0, T ; W^{1, \infty}(\Omega)\right)}\left\|\nabla \partial_{t} F_{h}\left(t^{\dagger}, \widetilde{Z}^{\dagger}\right)\right\|_{H^{k}(\Omega)}+\left\|\nabla F_{h}\right\|_{L^{\infty}([0, T] \times \Omega)}\left\|\partial_{t} F_{h}\left(t^{\dagger}, \widetilde{Z}^{\dagger}\right)\right\|_{H^{k}(\Omega)} \\
& +\left\|F_{h}\right\|_{L^{\infty}\left(0, T ; H^{3}(\Omega)\right)}\left\|\partial_{t} F_{h}\left(t^{\dagger}, \widetilde{Z}^{\dagger}\right)\right\|_{L^{2}(\Omega)}+\left\|F_{h}\right\|_{L^{\infty}([0, T] \times \Omega)}^{2}\left\|F_{h}\left(t^{\dagger}\right)\right\|_{H^{3}(\Omega)} \\
& +\left\|F_{h}\right\|_{L^{\infty}\left(0, T ; W^{1, \infty}(\Omega)\right)}^{2}\left\|F_{h}\left(t^{\dagger}\right)\right\|_{H^{2}(\Omega)}+\left\|F_{h}\right\|_{L^{\infty}\left(0, T ; W^{1, \infty}(\Omega)\right)}^{2}\left\|F_{h}\right\|_{H^{k}(\Omega)} \\
\leq & C\left(\sup _{n \leq N_{T}}\left\|f_{h}^{n}\right\|_{H^{3}(\Omega)}\right)\left(\left\|\nabla \partial_{t} F_{h}\left(t^{\dagger}, \widetilde{Z}^{\dagger}\right)\right\|_{H^{k}(\Omega)}+\left\|\partial_{t} F_{h}\left(t^{\dagger}, \widetilde{Z}^{\dagger}\right)\right\|_{H^{k}(\Omega)}+1\right) .
\end{aligned}
$$

The estimate of the term $\left\|\partial_{t} F_{h}\left(t^{\dagger}, \widetilde{Z}^{\dagger}\right)\right\|_{H^{k}(\Omega)}$ gives the same result as for the term $\left\|\partial_{t} F_{h}\left(t^{\natural}, \widetilde{Z}^{\natural}\right)\right\|_{H^{k}(\Omega)}$. We then have

$$
\begin{aligned}
\left\|\nabla \partial_{t} F_{h}\left(t^{\dagger}, \widetilde{Z}^{\dagger}\right)\right\|_{H^{k}(\Omega)} \leq & \frac{2\left\|\nabla F_{h}^{n+1 / 2}\left(\widetilde{Z}^{\dagger}\right)-\nabla F_{h}^{n}\left(\widetilde{Z}^{\dagger}\right)\right\|_{H^{k}(\Omega)}}{\Delta t}+\frac{2\left\|\nabla F_{h}^{n+1}\left(\widetilde{Z}^{\dagger}\right)-\nabla F_{h}^{n+1 / 2}\left(\widetilde{Z}^{\dagger}\right)\right\|_{H^{k}(\Omega)}}{\Delta t} \\
\leq & \frac{2\left\|\nabla F_{h}^{n}\left(\widetilde{Z}^{\dagger}\right)-\nabla F^{n}\left(\widetilde{Z}^{\dagger}\right)\right\|_{H^{k}(\Omega)}}{\Delta t} \frac{2\left\|\nabla F_{h}^{n+1}\left(\widetilde{Z}^{\dagger}\right)-\nabla F^{n+1}\left(\widetilde{Z}^{\dagger}\right)\right\|_{H^{k}(\Omega)}}{\Delta t} \\
& +\frac{4\left\|\nabla F_{h}^{n+1 / 2}\left(\widetilde{Z}^{\dagger}\right)-\nabla F^{n+1 / 2}\left(\widetilde{Z}^{\dagger}\right)\right\|_{H^{k}(\Omega)}+\frac{2\left\|\nabla F^{n+1 / 2}\left(\widetilde{Z}^{\dagger}\right)-\nabla F^{n}\left(\widetilde{Z}^{\dagger}\right)\right\|_{H^{k}(\Omega)}}{\Delta t}}{\Delta t} \\
& +\frac{2\left\|\nabla F^{n+1 / 2}\left(\widetilde{Z}^{\dagger}\right)-\nabla F^{n+1}\left(\widetilde{Z}^{\dagger}\right)\right\|_{H^{k}(\Omega)}}{\Delta t} .
\end{aligned}
$$


Therefore, using the inverse inequality (3.4), the approximation property (3.2) and assuming that $h / \Delta t$ is bounded, we get

$$
\begin{aligned}
\left\|\nabla \partial_{t} F_{h}\left(t^{\dagger}, \widetilde{Z}^{\dagger}\right)\right\|_{H^{k}(\Omega)} \leq & C\left\|\nabla \partial_{t} F\left(\cdot, \widetilde{Z}^{\dagger}\right)\right\|_{L^{\infty}\left(0, T ; H^{k}(\Omega)\right)}+\frac{C\left\|f_{h}^{n}-f^{n}\right\|_{H^{k+1}(\Omega)}}{\Delta t} \\
& +\frac{C\left\|f_{h}^{n+1 / 2}-f^{n+1 / 2}\right\|_{H^{k+1}(\Omega)}}{\Delta t}+\frac{C\left\|f_{h}^{n+1}-f^{n+1}\right\|_{H^{k+1}(\Omega)}}{\Delta t} \\
\leq & \frac{C\left\|f_{h}^{n}-f^{n}\right\|_{H^{k}(\Omega)}}{h \Delta t}+\frac{C\left\|f_{h}^{n+1 / 2}-f^{n+1 / 2}\right\|_{H^{k}(\Omega)}}{h \Delta t}+\frac{C\left\|f_{h}^{n+1}-f^{n+1}\right\|_{H^{k}(\Omega)}}{h \Delta t}+C .
\end{aligned}
$$

\section{REFERENCES}

[1] R.P. Agarwal, Difference equations and inequalities, Monographs and Textbooks in pure and applied mathematics. Marcel Dekker, New York, USA (1992).

[2] H. Andréasson and G. Rein, A numerical investigation of stability states and critical phenomena for the spherically symmetric Einstein-Vlasov system. Class. Quant. Grav. 23 (2006) 3659-3677.

[3] F. Bastin and P. Laubin, Regular compactly supported wavelets in Sobolev spaces. Duke Math. J. 87 (1996) 481-508.

[4] M.L. Bégué, A. Ghizzo, P. Bertrand, E. Sonnendrücker and O. Coulaud, Two dimensional semi-Lagrangian Vlasov simulations of laser-plasma interaction in the relativistic regime. J. Plasma Phys. 62 (1999) 367-388.

[5] N. Besse, Convergence of a semi-Lagrangian scheme for the one-dimensional Vlasov-Poisson system. SIAM J. Numer. Anal. 42 (2004) 350-382.

[6] N. Besse, Convergence of a high-order semi-Lagrangian scheme with propagation of gradients for the Vlasov-Poisson system. SIAM J. Numer. Anal. 46 (2008) 639-670.

[7] N. Besse and P. Bertrand, Gyro-water-bag approch in nonlinear gyrokinetic turbulence. J. Comput. Phys. 228 (2009) 39733995 .

[8] N. Besse and M. Mehrenberger, Convergence of classes of high-order semi-Lagrangian schemes for the Vlasov-Poisson system. Math. Comp. $\mathbf{7 7}$ (2008) 93-123.

[9] N. Besse and E. Sonnendrücker, Semi-Lagrangian schemes for the Vlasov equation on an unstructured mesh of phase space. J. Comput. Phys. 191 (2003) 341-376.

[10] N. Besse, G. Latu, A. Ghizzo, E. Sonnendrücker and P. Bertrand, A Wavelet-MRA-based adaptive semi-Lagrangian method for the relativistic Vlasov-Maxwell system. J. Comput. Phys. 227 (2008) 7889-7916.

[11] C.K. Birdsall and A.B. Langdon, Plasmas physics via computer simulation. McGraw-Hill, USA (1985).

[12] C.Z. Cheng and G. Knorr, The integration of the Vlasov equation in configuration space. J. Comput Phys. 22 (1976) 330-351.

[13] M.W. Choptuik, Universality and scaling in gravitational collapse of a scalar field. Phys. Rev. Lett. 70 (1993) 9-12.

[14] M.W. Choptuik and I. Obarrieta, Critical phenomena at the threshold of black hole formation for collisionless matter in spherical symmetry. Phys. Rev. D 65 (2001) 024007.

[15] M.W. Choptuik, T. Chmaj and P. Bizoń, Critical behaviour in gravitational collapse of a Yang-Mills field. Phys. Rev. Lett. 77 (1996) 424-427.

[16] Y. Choquet-Bruhat, Problème de Cauchy pour le système intégro-différentiel d'Einstein-Liouville. Ann. Inst. Fourier 21 (1971) 181-201.

[17] A. Cohen, Numerical analysis of wavelet methods, Studies in mathematics and its applications 32. Elsevier, North-Holland (2003).

[18] J.M. Dawson, Particle simulation of plasmas. Rev. Modern Phys. 55 (1983) 403-447.

[19] K. Ganguly and H. Victory, On the convergence for particle methods for multidimensional Vlasov-Poisson systems. SIAM J. Numer. Anal. 26 (1989) 249-288.

[20] R.T. Glassey and J. Schaeffer, Convergence of a particle method for the relativistic Vlasov-Maxwell system. SIAM J. Numer. Anal. 28 (1991) 1-25.

[21] G. Rein and A.D. Rendall, Global existence of solutions of the spherically symmetric Vlasov-Einstein with small initial data. Commun. Math. Phys. 150 (1992) 561-583. [Erratum. Comm. Math. Phys. 176 (1996) 475-478.]

[22] G. Rein and T. Rodewis, Convergence of a Particle-In-Cell scheme for the spherically symmetric Vlasov-Einstein system. Ind. Un. Math. J. 52 (2003) 821-861.

[23] G. Rein, A.D. Rendall and J. Schaeffer, A regularity theorem for solutions of the spherical symmetric Vlasov-Einstein system. Commun. Math. Phys. 168 (1995) 467-478. 
[24] G. Rein, A.D. Rendall and J. Schaeffer, Critical collapse of collisionless matter-a numerical investigation. Phys. Rev. D 58 (1998) 044007.

[25] T. Rodewis, Numerical treatment of the symmetric Vlasov-Poisson and Vlasov-Einstein system by particle methods. Ph.D. Thesis, Mathematisches Institut der Ludwig-Maximilians-Universität München, Munich, Germany (1999).

[26] J. Schaeffer, Discrete approximation of the Poisson-Vlasov system. Quart. Appl. Math. 45 (1987) 59-73.

[27] S.L. Shapiro and S.A. Teukolsky, Relativistic stellar dynamics on computer I, Motivation and numerical methods. Astrophys. J. 298 (1985) 34-57.

[28] S.L. Shapiro and S.A. Teukolsky, Relativistic stellar dynamics on computer II, Physical applications. Astrophys. J. 298 (1985) $58-79$.

[29] S.L. Shapiro and S.A. Teukolsky, Relativistic stellar dynamics on computer IV, Collapse of a stellar cluster to a black hole. Astrophys. J. 307 (1986) 575-592.

[30] A. Staniforth and J. Cote, Semi-Lagrangian integration schemes for atmospheric models-a review. Mon. Weather Rev. 119 (1991) 2206-2223.

[31] H.D. Victory and E.J. Allen, The convergence theory of particle-in-cell methods for multi-dimensional Vlasov-Poisson systems. SIAM J. Numer. Anal. 28 (1991) 1207-1241.

[32] H.D. Victory, G. Tucker and K. Ganguly, The convergence analysis of fully discretized particle methods for solving VlasovPoisson systems. SIAM J. Numer. Anal. 28 (1991) 955-989. 